\title{
Modeling Topological Relationships between Fuzzy Spatio-Temporal Objects
}

\author{
Haitao Cheng and $\mathrm{Fu}$ Zhang \\ School of Computer Science and Engineering, Northeastern University, Shenyang, China
}

Topological relationships between spatio-temporal objects are the most fundamental elements in spatio-temporal database systems, GIS, and image database systems. The research issue of modeling topological relationships has increasingly attracted attention, especially for querying of spatio-temporal objects and reasoning of topological relationships. Currently, topological relationship operating on spatio-temporal objects with precisely defined boundaries has been well studied. However, in the real world, spatio-temporal objects are not always crisp but with the nature of fuzziness and imprecision. Therefore, how to model topological relationship between fuzzy spatio-temporal objects is a significant topic and needs more investigations. This paper presents a study on modeling topological relationships between fuzzy spatio-temporal objects. Firstly, we give a model of fuzzy spatio-temporal objects in three-dimensional space and define those objects as moving fuzzy points, moving fuzzy lines, and moving fuzzy regions. On this basis, we propose a model for identifying basic topological relations between fuzzy spatio-temporal objects. Furthermore, in order to describe the evolution of basic topological relations over time, we give a model of complex topological relationships which are the sequences of basic relationships. The benefit of this model is that the complex topological relationships can be used as fuzzy spatio-temporal query operators in query languages. Finally, we provide some query examples to demonstrate fuzzy spatio-temporal queries in spatio-temporal database.

ACMCCS (2012) Classification: Computing methodologies $\rightarrow$ Artificial intelligence $\rightarrow$ Knowledge representation and reasoning $\rightarrow$ Vagueness and fuzzy logic Computing methodologies $\rightarrow$ Artificial intelligence $\rightarrow$ Knowledge representation and reasoning $\rightarrow$ Temporal reasoning

Computing methodologies $\rightarrow$ Artificial intelligence $\rightarrow$ Knowledge representation and reasoning $\rightarrow$ Spatial and physical reasoning

Computing methodologies $\rightarrow$ Modeling and simulation $\rightarrow$ Model development and analysis $\rightarrow$ Modeling methodologies

Keywords: topological relationships, fuzzy spatio-temporal object, modeling, query

\section{Introduction}

In the real world, time and space are closely related. By combining these two phenomena, an important research domain called spatio-temporal modeling emerges. Currently, modeling crisp spatio-temporal objects and their topological relationships is a topic of great importance and interest in the area of Geographical Information Systems (GIS), spatio-temporal database systems, Artificial Intelligence (AI), image database systems, and robotics [1]. Particularly, spatio-temporal topological relationships have been proved to be very important in these applications [2] because they can be employed to construct spatio-temporal database and support queries of spatio-temporal objects [3].

In modeling crisp spatio-temporal objects, two common approaches have been widely used: discrete model [4], [5], [6], [7] and continuous model [2], [8], [9]. The discrete model considers time as the third geometric dimension and represents discrete changes of spatial objects over time, while the continuous model represents continuous changes of spatial objects over time. Also, some approaches of modeling topological relationships between spatio-temporal objects have been proposed such as event-based method [10], an algebraic model [2], and space-time combination method [1]. All the approaches are based on the assumption that spatio-temporal objects have crisp boundaries and their positions can be accurately measured.

In fact, in the real world, spatio-temporal objects are not always crisp but with the nature of fuzziness and imprecision [11]. When spatio-temporal objects are fuzzy, the issue of fuzzy topological relationships emerges [12]. In meteorological system, for example, the bounda- 
ries of a foggy region and a windy region are fuzzy concepts and their positions change over time. A foggy region is disjoint with a windy region at the beginning, and later the fuzzy boundaries of the two regions meet, and finally the two regions are disjoint again. During this period, the topological relationships between both regions can be described as an evolution of basic topological relations (disjoint, fuzzy meet, and disjoint) over time. As a result, the issue of modeling topological relationships between fuzzy spatio-temporal objects should include two problems: ( $i$ ) modeling fuzzy spatio-temporal objects and (ii) modeling their topological relationships.

Currently, several efforts have been made for the representation of fuzzy spatio-temporal objects such as [13], [14], [15]. The approach in [13] focuses on a discrete change of the fuzzy region over time, but does not represent the continuous changes. In [14], a moving vague "Egg/Yolk" region is presented. The two approaches are limited to fuzzy region objects and do not take fuzzy point objects and fuzzy line objects into account. In [15], the authors only discuss spatio-temporal indeterminacy of moving fuzzy point objects. Temporal changes of fuzzy line and fuzzy region are missing. At the same time, in order to model fuzzy topological relationships between fuzzy spatio-temporal objects, some efficient approaches have been proposed, mainly including fuzzy set theory-based methods [15], [16], rough set theory-based method [17], point-set topology [13], motion-based approaches [14], [18], [19], and dimensional-extended method [20], [21]. For example, in [17], the authors propose a method to model spatio-temporal topological relationships based on rough set theory. In [19], the authors propose an approach for modeling spatio-temporal relations based on fuzzy logic. In reality, however, the described methods do not represent continuous change of these relationships. In [20], by extending the two-dimensional Egg/Yolk model into three-dimensional space, the authors obtain 92 spatio-temporal topological relations between fuzzy spatio-temporal regions over time. For each relation, the authors give graphical representation and the corresponding intersection matrix. However, the authors do not present the evolution of topological relations over time. In addition, the spatio-temporal relations in [17], [19], [20] are only defined in fuzzy regions, and not in fuzzy points and fuzzy lines. It should be noted that, although there have been some proposals for modeling topological relationships between fuzzy spatio-temporal objects, relatively little work has been done on integrating these topological relationships into databases to achieve fuzzy spatio-temporal queries. The detailed introduction about spatio-temporal objects and their topological relationships can be found in Section 2 of this paper.

The motivation for our research is the development of topological relationships model that allows identification of all topological relationships between fuzzy spatio-temporal objects and the query of fuzzy spatio-temporal objects through topological relationships. In this paper, we investigate a model of topological relationships between fuzzy spatio-temporal objects. The main components of the model are fuzzy spatio-temporal objects, basic topological relationships, and complex topological relationships. Fuzzy spatio-temporal objects, as the operands of fuzzy spatio-temporal topological relationships, are a widespread spatio-temporal phenomenon. The complex fuzzy topological relationships can express facts which are temporal evolutions of basic topological relations. It should be pointed out that topological relationships between fuzzy spatio-temporal objects are the basis of constructing fuzzy spatio-temporal description logic which will be studied in future work. The paper makes the following main contributions:

- We propose a model of fuzzy spatio-temporal objects. In our model, we define these objects as moving fuzzy points, moving fuzzy lines, and moving fuzzy regions. Fuzzy spatio-temporal objects, as operands of fuzzy spatio-temporal topological relationships, describe continuous movement of fuzzy spatial data types. Then, we give the graphical representation of fuzzy spatio-temporal objects. We also provide an application scenario of fuzzy spatio-temporal objects.

- We propose a model of basic topological relationships between fuzzy spatio-temporal objects. The modeling process includes four steps: $(i)$ establishing a new 9-intersection model based on the $3 \times 3$-intersection matrix presented in [22], (ii) identifying topological relationships by the matrix model, (iii) defining basic relations according to the intuitional meaning of topological relationships, and (iv) grouping topological relationships identified into basic relations. 
- Based on the proposed basic topological relationships, we model complex topological relationships between fuzzy spatio-temporal objects in order to describe the evolution of basic topological relations over time. More precisely, we firstly give the formal definition of complex fuzzy spatio-temporal relationships. Then, we elaborate specification of those relationships in the aspects of definition and graphical representation.

- We show how to use basic and complex topological relationships to query fuzzy spatio-temporal objects in the context of spatio-temporal databases.

The remainder of this paper is organized as follows. Section 2 contains a summary of the related work. Section 3 introduces some preliminaries about fuzzy set theory, spatial topological relations and temporal types, as far as they are relevant for this paper. Section 4 summarizes fuzzy spatial data types and defines fuzzy spatio-temporal objects, which represent the change of fuzzy spatial data types over time. In Section 4, we model basic fuzzy spatio-temporal relationships which describe the change of fuzzy spatial topological relationships over time. Section 5 describes how to model complex fuzzy spatio-temporal relationships based on basic relationships. In Section 6, we show some examples of fuzzy spatio-temporal queries. The last section concludes the paper with the summary and possible future studies.

\section{Related Work}

In this section, we summarize crisp spatio-temporal objects and their topological relationships (Section 2.1) and fuzzy spatio-temporal objects and their topological relationships (Section 2.2) in the literature.

\subsection{Crisp Spatio-Temporal Objects and their Topological Relationships}

The premise of topological relationships modeling is how to model fuzzy spatio-temporal objects. In modeling crisp spatio-temporal objects, two common approaches have been widely used: discrete model [4], [5], [6], [7] and continuous model [2], [8], [9].
In [7], the author defines a spatio-temporal object as a unified object that extends time to a spatial data model. The spatial objects mainly consider simplicial complexes in Euclidean two-dimensional space and the temporal information is attached to all components of the spatial objects. In [6], an entity with the character of motion can be defined as a spatio-temporal object. The motion can be described by a series of parameters, such as trajectory, speed, current location and duration of event. For instance, a spatio-temporal object called car can be described as car.Speed $=$ fast , car.Duration $=30$ minutes, car.Location $=\langle 0,5\rangle$. In [4], from the perspective of a discrete modeling, a spatio-temporal object $o$ is defined as a time-evolving spatial object. The development of the object $o$ can be represented by a set of triples $\left(o \_i d, s_{i}, t_{i}\right)$, where $o \_i d$ is an identification number of spatio-temporal object and $s_{i}$ is the position of the object at instant $t_{i}$. Grumbach and Rigaux [5] present a spatio-temporal data model based on linear constraints with the aim of representing infinite collections of points in 2-dimensional spaces. The trajectory of a spatio-temporal object can be considered as a linear constraint relation. All these approaches consider time as the third geometric dimension based on spatial objects. In addition, these spatio-temporal objects defined can only represent discrete changes of spatial objects over time. But these objects are incapable of representing the continuous temporal changes of spatial objects.

In order to establish a continuous spatio-temporal data model of time, Muller [9] defines a continuous spatio-temporal object called spatio-temporal region, which is considered as a primitive entity for representation of mereo-topological model. Erwig and Schneider [2] apply the temporal function $\tau(\alpha)=$ time $\rightarrow \alpha$ to the spatial data types $\alpha=\{$ point, line, region $\}$ and then yield three spatio-temporal data types $\tau($ point $)=$ moving point,$\tau($ line $)=$ evolving line, and $\tau($ region $)=$ evolving region. That is, spatio-temporal objects can be represented in three spatio-temporal data types. From a semantic point of view, a moving point is a point that changes its position in the Euclidean space over time. An evolving line is a line that can move and/or grow/shrink and/or change its linear structure. In the same way, an evolving region is a region that can move and/or grow/shrink and/or change its areal extent. In the fields of GIS and traffic planning navigation [8], moving 
objects such as cars, hurricanes and animals can also be considered as spatio-temporal objects. Take car and hurricane for example, they can be represented by moving points and moving regions respectively. In [8], the situation that the exact location of these objects changes over time can be taken into account. In fact, the approaches aiming at the movement of objects in [8], [9] are different from the approaches which focus on the shape change and movement of the objects over time in [2].

Spatio-temporal topological relationship is one of the most important and fundamental elements in spatio-temporal applications [20]. It plays an important role in the modeling of spatio-temporal knowledge. Topological relationships can be used for querying and reasoning spatio-temporal objects [23]. Some approaches of modeling topological relationships between spatio-temporal objects have been proposed such as an algebraic model [2], event-based method [10], and space-time combination approach [1].

In [2], Erwig and Schneider propose an explicit framework called spatio-temporal predicates for the representation of spatio-temporal knowledge. The formal definition of these predicates is based on three kinds of spatio-temporal objects: moving point, evolving line, and evolving region. Spatial predicates and sequence of elementary spatio-temporal predicates can be combined into complex predicates with the aim of describing the development of spatio-temporal relations between objects. In addition, all relationships can be extended to SQL language in order to achieve spatio-temporal queries [3]. In [10], the authors propose a model to analyze the spatio-temporal relationships between moving robots in RoboCup soccer game. This model is based on time intervals of continuous object motion (OMI) and duration of spatial relations (SRI), in which the authors used 13 Allen's time interval relations to describe temporal relationships and employed the quantitative direction and distance relations to describe spatial relations. In addition, according to the model, some spatio-temporal topological relationships such as meet, approaching, departing, and passing can be defined to interpret the behaviors of moving objects. The described methods, however, do not provide graphical representations for every basic spatio-temporal relation and do not demonstrate some query examples and only apply to the predicates that occur between moving point and evolving region as well as between moving points. Ribaric and Hrkac [1] propose a formal model for representing and reasoning spatio-temporal knowledge. This model describes a total of 117 spatio-temporal relationships, which are based on the combination of 13 Allen's time interval relations and 9 spatial relations. For example, the spatio-temporal relationship " $A \mathrm{ra}=B$ " means that the object $A$ is to the right and above the object $B$ and their activities are simultaneous. Unfortunately, these 117 relations can only express the relations between points and do not take into account the continuous change of spatio-temporal objects over time.

All of the above described approaches are based on the assumption that spatio-temporal objects have crisp boundaries and do not take into account the fuzzy boundaries of spatio-temporal objects, which can be a drawback when representing fuzzy and imprecise objects and their topological relationships in real-world applications.

Based on the above discussions, Table 1 summaries major work in modeling crisp spatio-temporal objects and their topological relationships.

\subsection{Fuzzy Spatio-Temporal Objects and their Topological Relationships}

Spatio-temporal objects are not always crisp, but fuzzy and uncertain in the real world [11]. In order to deal with the fuzziness and imprecision of spatio-temporal objects, several efforts have been made to model fuzzy spatio-temporal objects and their topological relationships, such as fuzzy set theory-based methods [15], [16], rough set theory-based method [17], point-set topology [13], motion-based approaches [14], [18], [19], and dimensional-extended method [20], [21].

There are several efforts to model spatio-temporal objects using fuzzy set theory [15], [16]. Pfoser and Jensen [15] represent a moving point object by using fuzzy techniques. Due to the measure error and sampling error, the object is imprecise. For example, the positions of a point object at a certain time or the positions of in-between point objects in time are imprecise. In [16], the authors model the fuzzy spatial and temporal information by using triangular fuzzy 
Table 1. Modeling crisp spatio-temporal objects and their topological relationships.

\begin{tabular}{|c|c|}
\hline Modeling crisp spatio-temporal objects & Comments \\
\hline $\begin{array}{l}\text { discrete model (Brisaboa, Mirbel and Pernici [6]; Grum- } \\
\text { bach, Rigaux and Segoufin [5]; Theodoridis et al. [4]; } \\
\text { Worboys [7]) }\end{array}$ & $\begin{array}{l}\text { representing discrete changes of spatial objects over } \\
\text { time }\end{array}$ \\
\hline $\begin{array}{l}\text { continuous model (Erwig and Schneider [2]; Muller [9]; } \\
\text { Schneider [8]) }\end{array}$ & $\begin{array}{l}\text { representing the continuous temporal changes of } \\
\text { spatial objects }\end{array}$ \\
\hline $\begin{array}{l}\text { Modeling topological relations between crisp spatio-tem- } \\
\text { poral objects }\end{array}$ & Major focuses \\
\hline algebraic model (Erwig and Schneider [2]) & $\begin{array}{l}\text { Spatio-temporal predicates based on moving point, } \\
\text { evolving line, and evolving region. }\end{array}$ \\
\hline event-based method (Miene and Visser [10]) & $\begin{array}{l}\text { spatio-temporal topological relationships between } \\
\text { moving points }\end{array}$ \\
\hline space-time combination approach (Ribaric and Hrkac [1]) & $\begin{array}{l}117 \text { spatio-temporal relationships based on the com- } \\
\text { bination of } 13 \text { Allen's time interval relations and } 9 \\
\text { spatial relations between points. }\end{array}$ \\
\hline
\end{tabular}

numbers, and obtain a total of 117 spatio-temporal relationships based on the combination of 13 temporal relationships and 9 spatial relationships. However, the described approaches only operate on fuzzy point objects and do not take fuzzy line and fuzzy region into account. In addition to fuzzy set, rough set can also be used for modeling spatio-temporal relationships [17]. In [17], Bassiri and Alesheikh propose a method to model topological relationships between spatio-temporal objects based on rough set. The method divides a rough spatial object into three areas including lower bound, boundary region, and outside region. Based on these areas, a 9-intersection matrix is obtained, which represents spatial relationships between two rough objects. With the combination of spatial relations and 13 Allen's time interval relations, the spatio-temporal relations can be generated.

An interesting approach for fuzzy spatio-temporal objects modeling in fuzzy topological space is given in [13]. Here, a land change is defined as a fuzzy spatio-temporal object. Based on the fuzzy topology, every object has a maximum of 5 areas, including interior, boundary of the boundary, interior of the boundary, boundary of the interior, and exterior. A traditional 9-intersection matrix can also be extended into 25 -intersection one. By comprising the changes of the five areas between temporal begin point and temporal end point, the situation of land use and land covers can be analysed. The approach in [13] only helps to describe a discrete change of fuzzy region over time, but does not explain the continuous changes. The temporal changes of fuzzy point and fuzzy line are also missing.
Some motion-based spatio-temporal relationships modeling approaches have also been proposed. In [14], the authors extend Muller's spatio-temporal theory to vague $\mathrm{Egg} /$ Yolk regions. A spatio-temporal relationship is also described as the spatial motion of vague Egg/Yolk regions over time. As a result, the model constructs some motion classes, such as POSSIBLE-HIT, POSSIBLE-REACH, and POSSIBLE-CROSS. In [19], an approach for modeling spatio-temporal relations based on fuzzy logic is proposed. In order to interpret an entity's behavior (e.g. a human) qualitatively in real-time, the approach designs four spatio-temporal relationships such as IsMoving, IsComingCloseTo, IsGoingAway, and IsGoingAlong. In [18], the authors use MBR (Minimum Bounding Rectangle) to represent fuzzy spatio-temporal position. A total of 6 spatio-temporal topological relationships are also presented, which include spatio-temporal disjoint, meet, intersect, contain, overlap, and equal.

Some work addresses an extension of two-dimensional Egg/Yolk model. In [20], by extending two-dimensional Egg/Yolk model to three-dimensional space, 92 topological relations between fuzzy spatio-temporal regions over time are obtained. For each relation, the authors give graphical representation and the corresponding intersection matrix. This model does not address the evolution of topological relationships and does not cover the motion. In [21], a model for indeterminate evolving $2 \mathrm{D}$ regions is proposed. The model extends the two-dimensional Egg/Yolk model into the third 
dimension that can describe the approximate topological relations. However, the spatio-temporal relations in [20], [21] are only defined in fuzzy regions, and not in fuzzy points and fuzzy lines.

It should be noted that, although some efficient approaches have been proposed for modeling fuzzy topological relationships between fuzzy spatio-temporal objects, relatively little work has been done on integrating these fuzzy topological relationships into spatio-temporal databases to query fuzzy spatio-temporal objects.

Based on the above discussion, Table 2 presents a summary of various fuzzy spatio-temporal objects and their topological relationships models proposed in the literature.

In this paper, we introduce fuzzy set theory and fuzzy spatial objects to the model represented in [2] for modeling fuzzy spatio-temporal objects. It is necessary to construct a generic fuzzy spatio-temporal objects model covering the discrete and continuous temporal change of moving fuzzy point, moving fuzzy line and moving fuzzy region. In addition, fuzzy spatio-temporal objects are the basis of modeling fuzzy spatio-temporal topological relationships. We also use a 9-intersection model and motion properties to model basic and complex topological relationships between fuzzy spatio-temporal objects. In particular, these topological relationships can be considered as fuzzy spatio-temporal operators in order to achieve fuzzy spatio-temporal queries in this paper.

\section{Preliminaries}

In this section, we briefly recall some preliminaries. Section 3.1 introduces fuzzy set theory, and Section 3.2 presents the concept of spatial topological relations. Section 3.3 introduces temporal types.

\subsection{Fuzzy Set Theory}

In order to model imprecise and fuzzy information in the real-world applications, Zadeh [24] proposes the theory of fuzzy sets. As an extension of classical crisp set, fuzzy set theory is widely applied in various domains such as information systems, artificial intelligence, expert systems, computer science and Semantic Web [25]. Here, we only briefly introduce the basic definition, representation, and operations of fuzzy set theory.

Let $U$ be a universe of discourse, the fuzzy set $F$ in $U$ is characterized by a membership function $\mu_{F}(u): U \rightarrow[0,1]$, which assigns a real number in the interval $[0,1]$ to each $u$ in $U$. For each

Table 2. Comparison of topological relationships models between fuzzy spatio-temporal objects.

\begin{tabular}{|c||c|c|c|c|c|c|c|c|}
\hline $\begin{array}{c}\text { Corresponding } \\
\text { reference }\end{array}$ & $\begin{array}{c}\text { fuzzy set } \\
\text { based }\end{array}$ & $\begin{array}{c}\text { rough set } \\
\text { based }\end{array}$ & $\begin{array}{c}\text { point-set } \\
\text { topology }\end{array}$ & $\begin{array}{c}\text { motion- } \\
\text {-based }\end{array}$ & $\begin{array}{c}\text { dimen- } \\
\text { sional- } \\
\text {-extended }\end{array}$ & $\begin{array}{c}\text { fuzzy } \\
\text { point }\end{array}$ & fuzzy line & $\begin{array}{c}\text { fuzzy } \\
\text { region }\end{array}$ \\
\hline \hline $\begin{array}{c}\text { Pfoser and } \\
\text { Jensen [15] }\end{array}$ & $\sqrt{ }$ & & & & & $\sqrt{ }$ & & \\
\hline $\begin{array}{c}\text { Ribaric and } \\
\text { Hrkac [16] }\end{array}$ & $\sqrt{ }$ & & & & & $\sqrt{ }$ & \\
\hline $\begin{array}{c}\text { Bassiri and } \\
\text { Alesheikh [17] }\end{array}$ & & $\sqrt{ }$ & & & & & & $\sqrt{ }$ \\
\hline Bassiri et al. [13] & & & $\sqrt{ }$ & & & & & $\sqrt{ }$ \\
\hline Bai et al. [18] & & & & $\sqrt{ }$ & & & & $\sqrt{ }$ \\
\hline $\begin{array}{c}\text { Ibrahim and } \\
\text { Tawfik [14] }\end{array}$ & & & & $\sqrt{ }$ & & & & $\sqrt{ }$ \\
\hline $\begin{array}{c}\text { Le Yaouanc and } \\
\text { Poli [21] }\end{array}$ & & & & $\sqrt{ }$ & & $\sqrt{ }$ & \\
\hline Bai et al. [20] & & & & & $\sqrt{ }$ & & $\sqrt{ }$ \\
\hline Lei et al. [21] & & & & & $\sqrt{ }$ & & \\
\hline Our work & $\sqrt{ }$ & & & $\sqrt{ }$ & & $\sqrt{ }$ \\
\hline
\end{tabular}


$u \in U$, the value of $\mu_{F}(u)$ at $u$ represents the degree of membership of $u$ in $U$. For example, $\mu_{F}(u)=0.5$ means that $u$ belongs to $F$ with a degree of 0.5 .

For the basic operations, we assume that let $A$ and $B$ be two fuzzy sets over $U$. For any $u \in U$, fuzzy sets $A$ and $B$ defined by $U$, membership function comprises three kinds of constraints as follows [24]:

$$
\begin{aligned}
\mu_{A \cup B}(u) & =\mu_{A}(u) \vee \mu_{B}(u) \\
\mu_{A \cap B}(u) & =\mu_{A}(u) \wedge \mu_{B}(u) \\
\mu_{\bar{A}}(u) & =1-\mu_{A},
\end{aligned}
$$

where $\bar{A}$ is complement of $A$ in $U$.

\subsection{Spatial Topological Relations}

As mentioned in [26], spatial relations include three classes, such as topological, metric and directional relations. In our work, we only discuss the topological spatio-temporal relations. Thus, in this section, we omit the introduction of metric and directional relations.

Spatial topological relations play a significant role in Geographic Information System and spatial database, whose purpose is to describe relationships between crisp spatial objects [11]. Randell et al. [27] propose the Region Connection Calculus (short for RCC) theory in the field of spatial domain knowledge. And the most important model in RCC is the RCC-8, which represents 8 kinds of $\mathrm{RCC}$ relationships, i.e., $\{D C$, $E C, E Q, N T P P, N T P P i, T P P, T P P i, P O\}$, and the corresponding semantics are disjoint, meet, equal, contains, inside, covers, coveredby, and overlap, respectively. Currently, the most widely used model for analyzing topological relations is a 9-intersection model, which is based on the intersection between the parts (interior, boundary, exterior) of crisp spatial objects [28]. For two crisp spatial objects $A$ and $B$, let $A^{0}, \partial A$, $A^{-}$be the interior, boundary, and exterior of $A$, respectively. Similarly, let $B^{0}, \partial B, B^{-}$be the interior, boundary, and exterior of $B$, respectively. Then, a set of topological relations can be concisely represented by the following $3 \times 3$ matrix $R(A, B)$, named the 9 -intersection matrix.

$$
R(A, B)=\left[\begin{array}{lll}
A^{0} \cap \partial B & A^{0} \cap B^{0} & A^{0} \cap B^{-} \\
\partial A \cap \partial B & \partial A \cap B^{0} & \partial A \cap B^{-} \\
A^{-} \cap \partial B & A^{-} \cap B^{0} & A^{-} \cap B^{-}
\end{array}\right]
$$

The value of each intersection in the matrix contains either a 0 (empty) or a 1 (nonempty), resulting in $2^{9}=512$ possible topological relations. Only when the spatial objects are embedded in the dimensions of $R^{2}$ can the topological relations be realized [22]. By eliminating those relations that are not realized, one can obtain a total of 2 relations (disjoint and equal) between two crisp points, 3 relations (disjoint, meet, contains) between one crisp point and line, 3 relations (disjoint, meet, contains) between one crisp point and region, 16 relations between two crisp lines, 13 relations between one crisp line and region [28], 8 relations (disjoint, meet, equal, contains, inside, overlap, covers, and coveredby) between two crisp regions (see Figure 1).

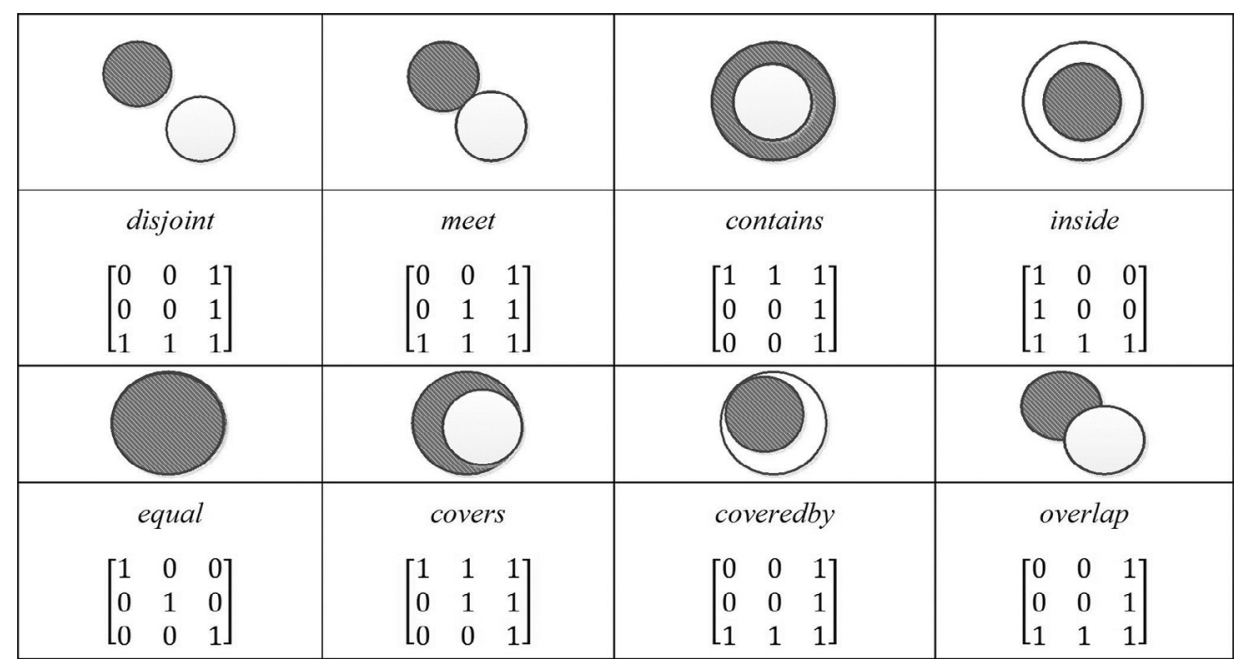

Figure 1. The eight relationships (predicates) between two crisp regions. 


\subsection{Temporal Types}

Time is an essential feature of the real-world, especially in a natural phenomenon. Any objects can change in a certain time point or time period. It is important to note that time can describe the continuity of objects in motion or the sequence of objects and processes. Generally, temporal types consist of three forms, time point, time interval, and time duration [29], [30], [31]. In the following, we will recall briefly the notions of temporal types.

Time point: Time point called instant is the discrete time when state of an object or relation between two objects holds. For example, a ship leaves a windy region at 5:30 am. The system of time point can be represented by a formal method. A system of time point $T=\left\langle t, \leq_{t}\right\rangle[29]$, where $t$ is a finite set of time points $t=\left\{t_{1}, t_{2}, \ldots, t_{n}\right\}, n \geq 0, \leq_{t}$ denotes the temporal order over $t$. For instance, $t_{1} \leq_{t} t_{2}$ denotes $t_{1}$ occurs before $t_{2}$ or $t_{1}$ and $t_{2}$ occur at the same time.

Time interval: The value of time interval is a collection of pairs of time points where the former is the start time point $t_{s}$ and the latter is the end one $t_{e}$. i.e., a time interval $T=\left[t_{s}, t_{e}\right]$. For example, a ship crosses a windy region with a time interval [4:30 am, 5:30 am]. It should be pointed out that some events hold only at a restricted time interval. This time interval called valid time is the intersection of two participating temporal objects's domains. For instance, a ship starts its journey at 1:00 am and finishes the journey at 9:00 am. Within the interval, a windy region is to appear and disappear at 3:00 am and 5:30 am, respectively. The valid time of the two objects' associated topological relationship is between 3:00 am to 5:30 am.

Time duration: Time duration indicates a sustained period of time, the value of which is a length of time. For example, consider an event that a storm lasted three hours. The time duration is three hours. A similarity can be observed between Time duration and Time interval - both of them represent a period of time. However, the most obvious difference is that Time duration has neither begin time nor end time.

\section{Modeling Fuzzy Spatio-Temporal Objects}

In order to model topological relationships between fuzzy spatio-temporal objects, the first problem is how to model fuzzy spatio-temporal objects because these objects are the basic operations of fuzzy topological relationships. In this section, we first review fuzzy spatial objects in Section 4.1, and then, taking it as a basis, we give the formal definition of fuzzy spatio-temproal objects called moving fuzzy points, moving fuzzy lines, and moving fuzzy regions in Section 4.2.

\subsection{Fuzzy Spatial Data Types}

In order to model fuzzy spatio-temporal objects, it is necessary for one to fully understand the basic spatial data types, such as points, lines, and regions. In terms of a crisp case, crisp points, lines, and regions have been widely discussed in [32]. In terms of fuzzy case, Liu et al. [12], Schneider [33], Pauly et al. [34] and Tang et al. [35] have proposed the definitions of fuzzy points, fuzzy lines and fuzzy regions. In the following, we summarize the definitions as follows.

Definition 1 (Fuzzy point). A fuzzy point is a point, and its exact location is unknown, but its possible locations may be in a certain area. Let $P$ be a fuzzy point. Then $P$ consists of three parts: a boundary $(\partial P)$, an exterior $\left(P^{-}\right)$, and an interior $\left(P^{0}\right)$, where $\partial P$ can be empty. For any $\partial P$, there is a membership function $\mu_{p}(x, y)$ such that $0<\mu_{p} \leq 1$, where $x$ and $y$ denote the $x$-coordinate and $y$-coordinate of fuzzy point $P$, respectively. An illustration of fuzzy point $P$ is shown in Figure 2.

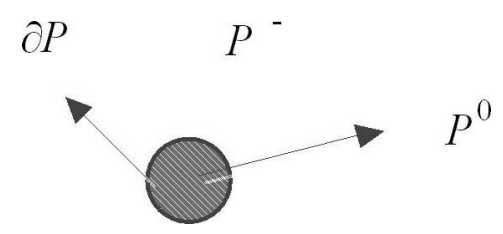

Figure 2. An illustration of a fuzzy point $P$.

Definition 2 (Fuzzy line). A fuzzy line is a line for which the exact shape, position or lengths are not known, but it is known in which area 
the line must be. Let $L$ be a fuzzy line, $\partial L$ be a boundary of $L, L^{-}$be an exterior of $L, L^{0}$ be an interior of $L$. For any $\partial L$, there is a membership function $\mu_{L}(x, y)$ such that $0<\mu_{L} \leq 1$, where $x$ and $y$ denote the $x$-coordinate and $y$-coordinate of fuzzy line $L$, respectively. An illustration of fuzzy line $L$ is shown in Figure 3.

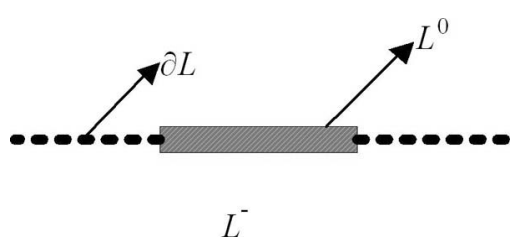

Figure 3. An illustration of a fuzzy line $L$.

Definition 3 (Fuzzy region). A fuzzy region $R$ is a region with fuzzy boundaries, which is made up of two crisp regions, $R_{d}$ and $R_{f}$, where $R_{d}$ describes the determinate part of $R$, and $R_{f}$ describes the fuzzy part of $A . \partial R_{d}$ and $\partial R_{f}$, representing the two fuzzy boundaries, are the inner and outer boundaries of $R$, respectively, where $\mu_{R_{d}}=1$, and $\mu_{R_{f}}=0$ describe the degrees of membership belonging to determinate part. It is important to note that the degree of membership decreases as the fuzzy part moves away from the determinate one. $R^{-}$is an exterior of the region $R$. A fuzzy region is illustrated in Figure 4 with the determinate $R_{d}$, the inner boundary $\partial R_{d}$, the outer boundary $\partial R_{f}$, and the exterior $R^{-}$.

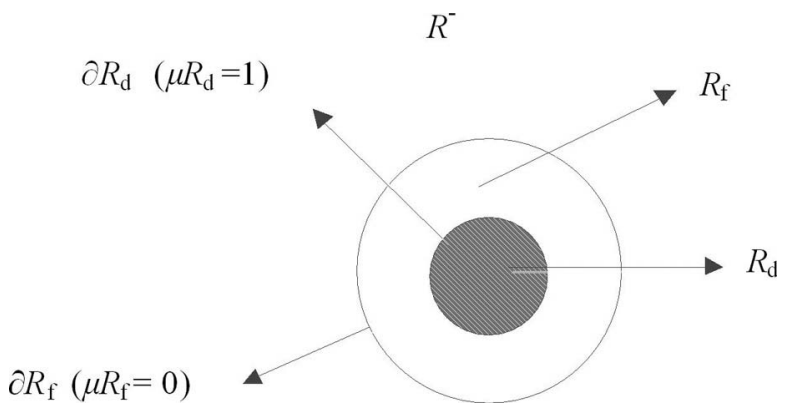

Figure 4. An illustration of a fuzzy region $R$ with fuzzy boundaries.

\subsection{Modeling of Fuzzy Spatio-temporal Objects}

Before modeling topological relationships between fuzzy spatio-temporal objects, we first need to model fuzzy spatio-temporal objects.
As mentioned in Section 4.1, fuzzy spatial objects (also called fuzzy spatial data types) are considered as static objects which include fuzzy points, fuzzy lines and fuzzy regions. Compared with them, fuzzy spatio-temporal objects are viewed as moving objects, the positions of which change over time. In other words, fuzzy spatio-temporal objects represent the change of fuzzy spatial objects over time.

Based on fuzzy spatial objects [12], [34], [35] and the representation of motion model [23], we first give a formal definition of motion.

Definition 4 (Motion). The motion of a moving objectisacontinuousfunctionoffuzzyspatio-temporal data types FST from fuzzy time ftime to fuzzy spatial data types $F S$ :

$$
\text { FST: ftime } \rightarrow F S,
$$

Here, $F S$ denotes fuzzy spatial data types including fuzzy points, fuzzy lines and fuzzy regions. FST represents all total functions from ftime to $F S$. The symbol of ftime represents fuzzy time with a degree of membership $\mu_{\text {time }} \rightarrow[0,1]$.

From the above definition, it is shown that fuzzy spatial objects change continuously over time. Fuzziness is mainly reflected in spatial data types and time. And the definition describes not only continuous change of fuzzy spatio-temporal objects, but also discrete change. If ftime denotes a time point, then the definition can describe a discrete change. If ftime denotes a time interval, then the definition can describe a continuous change.

Now, we define fuzzy spatio-temporal objects. Fuzzy spatio-temporal objects can be seen as fuzzy spatio-temporal data types which describe continuous movement of fuzzy spatial datatypes. As we have stated in Section 4.1, fuzzy spatial objects comprise fuzzy point, fuzzy line, and fuzzy region. Thus, we define fuzzy spatio-temporal objects as moving fuzzy points, moving fuzzy lines (or called evolving fuzzy lines), and moving fuzzy regions (or called evolving fuzzy regions). Based on the Definition 4, we give the formal definitions of the three kinds of fuzzy spatio-temporal objects.

Definition 5. Let mfpoint, mfline, and mfregion denote moving fuzzy points, moving fuzzy lines, and moving fuzzy regions. They are defined as follows: 


$$
\begin{aligned}
& \text { mfpoint }=\{F S T: \text { ftime } \rightarrow F S \mid \\
& \forall t \in \text { domain }(F S T), F S T(t) \in \text { fuzzy point }\} \\
& \text { mfline }=\{F S T: \text { ftime } \rightarrow F S \mid \\
& \forall t \in \text { domain }(F S T), F S T(t) \in \text { fuzzy line }\} \\
& \text { mfregion }=\{F S T: f t i m e \rightarrow F S \mid \\
& \forall t \in \text { domain }(F S T), F S T(t) \in \text { fuzzy region }\}
\end{aligned}
$$

From the definition above, we can see that fuzzy spatio-temporal objects describe continuous movement of fuzzy spatial objects. In order to well understand these fuzzy spatio-temporal objects, we give the following explanations.

- mfpoint: the positions of a fuzzy point change over time, which forms a fuzzy point motion trajectory. Take ships for example, a ship waiting in the queue for crossing "Qiongzhou Strait" can be represented by a moving fuzzy point since it is found at a certain point, but may change its position from time to time.

- mfline: the positions and routes of a fuzzy line change over time, which forms a fuzzy line motion trajectory. For example, a river can be represented by a moving fuzzy line objects (or called evolving fuzzy line object) since a river's changing line can change over floods and drought.

- mfregion: the positions of a fuzzy region change over time, which forms a fuzzy region motion trajectory. For instance, we view hurricane as a moving fuzzy region since its boundaries can be fuzzy and its positions also change over time.
Now, let us give the graphical representation of fuzzy spatio-temporal objects. In order to clearly represent the continuity of fuzzy spatio-temporal objects, we utilize a discrete representation called sliced presentation [36] which decomposes the temporal development of a value into many sections called "slices" such that within a slice this development of objects can be expressed by some simple function. Here, we take an example to illustrate the fuzzy spatio-temporal objects.

As shown in Figure 5, for a moving fuzzy region $O$, time is sliced into three sections denoted by $t_{1}, t_{2}$ and $t_{3}$, respectively $\left(t_{1}<t_{2}<t_{3}\right)$. The time can be fuzzy with a degree of membership denoted by $\mu_{t_{1}}, \mu_{t_{2}}$ and $\mu_{t_{2}}$. When time $=$ $t_{1}$, the position of $O$ is shown in the left graphic. The middle graphic shows the position of $O$ at time $=t_{2}$. Similarly, the right graphic illustrates the position of $O$ at time $=t_{3}$.

Derived from Figure 5, an illustration of a moving fuzzy region $O$ during time interval $I$ (from $t_{1}$ to $t_{3}$ ) is obtained in Figure 6, in which the time axis is regarded as the third geometric dimension. In other words, Figure 6 represents the trajectory of a fuzzy region $O$ during time interval $I$ (from $t_{1}$ to $t_{3}$ ). For reasons of space, we only give a graphical representation of a moving fuzzy region. For the graphical representation of the other fuzzy spatio-temporal objects, the method is the same as the one of moving fuzzy points. Here, we do not elaborate them in detail.

Next, let us consider an application scenario of modeling fuzzy spatio-temporal objects.
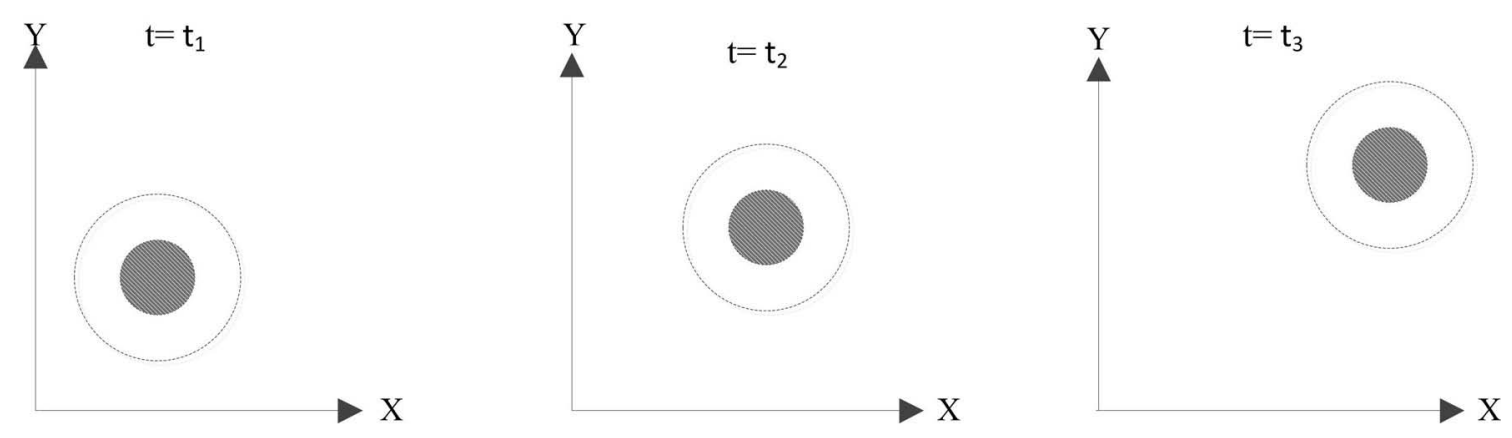

Figure 5. The position change of a moving fuzzy region $O$ :

(i) the left graphic shows the position at time $t_{1}$;

(ii) the middle graphic shows the position at time $t_{2}$;

(iii) the right graphic illustrates the position at time $=t_{3}$. 


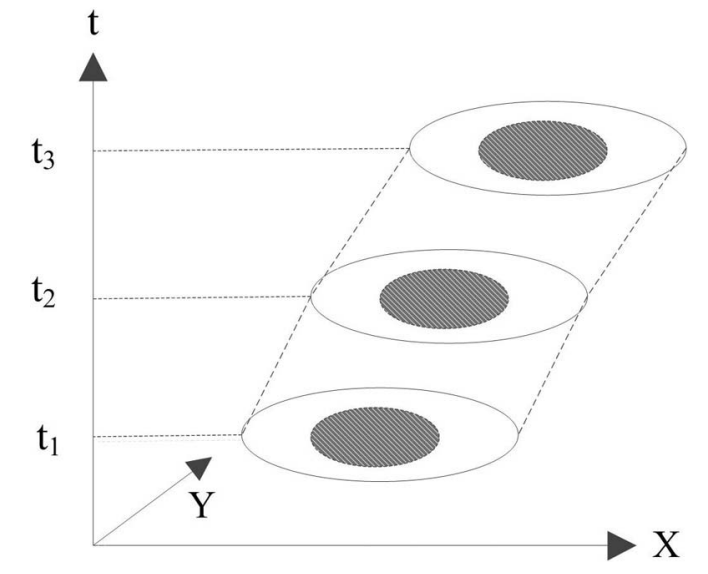

Figure 6. An illustration of a moving fuzzy region $O$ during one time peroid derived from Figure 5.

Example 1. Consider a discrete spatio-temporal phenomenon. The borders of a lake can change because of seasonal rainfall within a year. Seasonal changes affect the gray belt around the lake. The gray belt is a border. The borders of the lake are indeterminate since different season can have different amount of rainfall. Hence, the lake with imprecise borders can be abstracted to fuzzy spatio-temporal object.

Figure 7 shows the results of changes of the borders of a fuzzy lake object over season. Every color represents one border of the lake region at a certain season. For representation of fuzziness, every color has a membership value. As we have mentioned, the actual borders of the lake region can change position and shape due to the amount of rainfall in different seasons. The center region shows the minimum shape of a lake in winter and the borders have a membership value 1 . In summer, we assume that the borders have a membership value $[0,0.2]$. The example illustrates the modeling of fuzzy spatio-temporal objects.

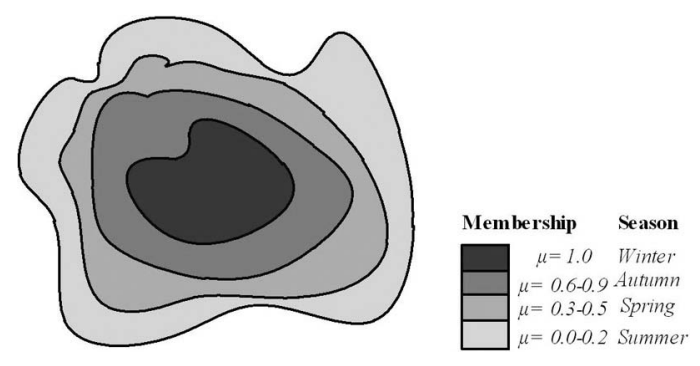

Figure 7. The changes of the borders of fuzzy lake region over season.
The model of fuzzy spatio-temporal objects not only describes discrete lake region, but it also represents a continuous spatio-temporal phenomenon. The most typical application is the representation of moving objects. For example, a moving car in city traffic that changes position over time can be represented as a moving fuzzy point. A moving typhoon can be abstracted to a moving fuzzy region since it changes position over time and its borders are also indeterminate. In addition, the meteorological phenomena can be described by fuzzy spatio-temporal objects. For instance, foggy regions, windy regions, and wavy regions can be abstracted to moving fuzzy regions.

It is important to point out that some fuzzy spatio-temporal objects are static or do not move during some time intervals. That is, the positions of some fuzzy points, fuzzy regions or fuzzy lines do not change over time. For example, one river or mountain does not change during a long peroid of time. Whether fuzzy spatio-temporal objects are dynamic or static, their topological relationships should be modeled.

Topological relationships between spatio-temporal objects are the most fundamental elements in many applications [20]. A major benefit of topological relationships is that they can be used for spatio-temporal query in databases and reasoning in knowledge bases. In the next section, we will present a modeling of topological relationships between fuzzy spatio-temporal objects. When fuzzy spatio-temporal objects are static or their topological relationships do not change during a time interval, we should model basic topological relationships which will be discussed in Section 5. When fuzzy spatio-temporal objects are dynamic, we should model complex topological relationships describing the evolution of basic topological relationships, which will be discussed in Section 6.

\section{Modeling Basic Topological Relationships}

In this section, we first propose a model of basic topological relationships between fuzzy spatio-temporal objects (see Section 5.1). Then, using the model, we identify topological relationships between two fuzzy spatio-temporal objects (see Section 5.2). 


\subsection{Formal Definition of Basic Topological Relationships}

In order to well understand fuzzy spatio-temporal relationships which will be defined in the following, we first discuss the time problem related to those relationships.

Suppose that $t_{1}$ and $t_{2}$ denote the time domains of fuzzy spatio-temporal objects $O_{1}$ and $O_{2}$, respectively. For the valid fuzzy time, we can analyze four situations: $(i) t_{1}$ and $t_{2}$ must be totally defined, which is a prerequisite of the fuzzy spatio-temporal relationships; ( $i i)$ if $t_{1} \cap t_{2}=\varnothing$, then the valid fuzzy time does not exist; (iii) if $t_{1} \cap t_{2} \neq \varnothing$, then the valid fuzzy time of fuzzy spatio-temporal relationship between $O_{1}$ and $O_{2}$ is the intersection of $t_{1}$ and $t_{2} ;(i v)$ if $t_{1} \subset t_{2}$, then the valid fuzzy time is $t_{1}$; $(v)$ if $t_{2} \subset t_{1}$, then the valid fuzzy time is $t_{2}$; Accordingly, we define the valid fuzzy time $t$ as the intersection of $t_{1}$ and $t_{2}$. Hence, in the perspective of fuzzy time of fuzzy spatio-temporal relationship, we only consider the valid fuzzy time of two fuzzy spatio-temporal objects. That is, we only research the fuzzy spatio-temporal topological relationships which hold at the same time interval or time point.

Now, we give the formal definition of basic topological relationships. As described in Section 4.1, a fuzzy spatial type (fuzzy spatial object) consists of a boundary, an exterior, and an interior. Hence, based on the $3 \times 3$-intersection matrix model presented in [22], we propose a new 9-intersection model for identifying basic topological relations between two fuzzy spatio-temporal objects.

Definition 6 (Fuzzy Spatio-temporal Relationships). Let $A, B$ denote two fuzzy spatio-temporal objects represented in Definition 5 and $\cap_{t}$ denote intersection during time interval $t$. Let $\partial A, A^{-}$, and $A^{0}\left(\partial B, B^{-}\right.$, and $\left.B^{0}\right)$ be a fuzzy part, exterior, determinate part of $A(B)$. Fuzzy spatio-temporal relationship $R$ between $A$ and $B$ during time interval $t$ is defined as:

$R(A, B, t)=\left[\begin{array}{lll}A^{0} \cap_{t} B^{0} & A^{0} \cap_{t} \partial B & A^{0} \cap_{t} B^{-} \\ \partial A \cap_{t} B^{0} & \partial A \cap_{t} \partial B & \partial A \cap_{t} B^{-} \\ A^{-} \cap_{t} B^{0} & A^{-} \cap_{t} \partial B & A^{-} \cap_{t} B^{-}\end{array}\right]$

It should be noted that every element of the $3 \times 3$-intersection matrix contains either a 0 or a 1 which denote whether that intersection $\left(\cap_{t}\right)$ is empty or non-empty respectively. $\cap_{t}$ indicates that the topological relationship holds during time interval $t$. In the following, we give the definition of $\bigcap_{t}$.

Definition 7. Given two fuzzy spatio-temporal objects $A$ and $B$, let $t_{A}$ and $t_{B}$ denote the time intervals of $A$ and $B$, respectively. Then the $\cap_{t}$ can be defined as:

$$
\begin{aligned}
A \cap_{t} B & =\left(\partial A \vee A^{-} \vee A^{0}\right) \cap_{t_{A} \cap t_{B}}\left(\partial B \vee B^{-} \vee B^{0}\right) \\
& \wedge A^{0}\left(t_{A} \cap t_{B}\right) \neq \varnothing \wedge B^{0}\left(t_{A} \cap t_{B}\right) \neq \varnothing
\end{aligned}
$$

By examining the elements of the 9-intersection model, we can identify topological relationships between two fuzzy spatio-temporal objects.

It should be noted that our proposed spatio-temporal topological relations cannot deal with the continuous transition of membership degrees of the fuzzy spatial objects. This problem will be studied in future work. Next, we will study the identification of topological relationships.

\subsection{Identification of Topological Relationships}

Based on the new 9-intersection model, a total of $2^{9}=512$ possible topological relations can be obtained. However, not all of the topological relations can be represented in the reality. Thus, we need to identify possible topological relationships. In the following, we first give the conditions which the above $3 \times 3$-intersection matrix must satisfy.

- each part of $A\left(\partial A, A^{-}\right.$, and $\left.A^{0}\right)$ must intersect with at least one part of $B\left(\partial B, B^{-}\right.$, and $\left.B^{0}\right)$, and vice versa.

- if $A^{0} \cap_{t} B^{0}$ and $A^{-} \cap_{t} B^{0}$ are 1 , then $\partial A \cap_{t} B^{0}$ must be 1 , and vice versa.

- if $A^{0} \cap_{t} \partial B$ and $\partial A \cap_{t} B^{0}$ are 1 , then $\partial A \cap_{t} \partial B$ must be 1 .

- if $A^{0} \cap_{t} B^{0}$ is 0 and $A^{0} \cap_{t} \partial B$ is 1 , then $\partial A \cap_{t} \partial B$ must be 1 .

- if $A^{0} \cap_{t} B^{0}$ and $\partial A \cap_{t} B^{0}$ are 1 , then $A^{-} \cap_{t} B^{0}$ must be 0 , and vice versa.

- if $A^{0} \cap_{t} \partial B$ and $A^{-} \cap_{t} \partial B$ are 1 , then $\partial A \cap_{t} \partial B$ must be 1 , and vice versa. 
Now, we discuss the identification of topologicalrelationshipsbetweentwo fuzzy spatio-temporal objects. It should be noted that, due to the limitations on the length of this paper, we only investigate topological relationships among two moving fuzzy points, one moving fuzzy point to one moving fuzzy region, and two moving fuzzy regions. The topological relationships among one moving fuzzy point to one moving fuzzy line, two moving fuzzy lines, and one moving fuzzy line to one moving fuzzy region will be studied and reported in a seperate paper.

In the following, we discuss the identification of topological relationships in the cases of mfpoint/mfpoint, mfpoint/mfregion, and mfregion/mfregion. Each case includes three steps: (i) identifying topological relationships by the above matrix, (ii) defining basic relations according to the intuitional meaning, and (iii) grouping topological relationships identified into basic relations.

\section{In the case of $m f$ point/mfpoint.}

Based on above conditions, by eliminating those relationships that are not realized, we can identify 3 different topological relations between two moving fuzzy points. The relationships between two moving fuzzy points are shown in Figure 8, and they include the value of matrix plus the support view of 3-dimensional spaces.

From the Figure 8, we can see that either two moving fuzzy points are always disjoint, or the two moving fuzzy points can always meet, or the two moving fuzzy points can meet possibly.
Due to the fuzziness of relationships, the existing spatial relationships (e.g., disjoint, meet) are no longer valid for these moving fuzzy points. According to the intuitional meaning of three topological relations we give the formal definition of three basic topological relationships between two moving fuzzy points.

Definition 8. Let $A$ and $B$ denote two moving fuzzy points, and let $t$ be a temporal point or a temporal interval. Time domains of $A$ and $B$ are given by $\operatorname{domain}(A)$ and $\operatorname{domain}(B)$, respectively. Fuzzy topological relationships between two moving fuzzy points are defined as follows.

$\operatorname{Disjoint}(A, B, t)=\forall t \in \operatorname{domain}(A) \cap \operatorname{domain}(B)$ :

$$
\begin{aligned}
A^{0} \cap_{t} B^{0} & =\varnothing \wedge A^{0} \cap_{t} \partial B=\varnothing \wedge \partial A \cap_{t} B^{0} \\
& =\varnothing \wedge \partial A \cap_{t} \partial B=\varnothing .
\end{aligned}
$$

$\operatorname{Meet}(A, B, t)=\forall t \in \operatorname{domain}(A) \cap \operatorname{domain}(B)$ :

$$
\begin{aligned}
A^{0} \cap_{t} \partial B^{0} & =\varnothing \wedge \partial A \cap_{t} B^{0}=\varnothing \wedge A^{0} \cap_{t} B^{-} \\
& =\varnothing \wedge A^{-} \cap_{t} B^{0}=\varnothing .
\end{aligned}
$$

$\boldsymbol{F P P M e e t}(A, B, t)=\exists t \in \operatorname{domain}(A) \cap \operatorname{domain}(B)$ : $\neg \operatorname{Disjoint}(A, B, t) \wedge \neg \operatorname{Meet}(A, B, t)$.

This definition means that there are 3 kinds of fuzzy topological relationships between two moving fuzzy points. The Disjoint means that two moving fuzzy points always disjoint each other during a time interval $t$ or at a time point $t$. Similarly, Meet indicates that two moving fuzzy points always meet each other. Besides, the FPPMeet is used to deal with this case that two moving fuzzy points can meet with a degree during a time interval or at a time point.

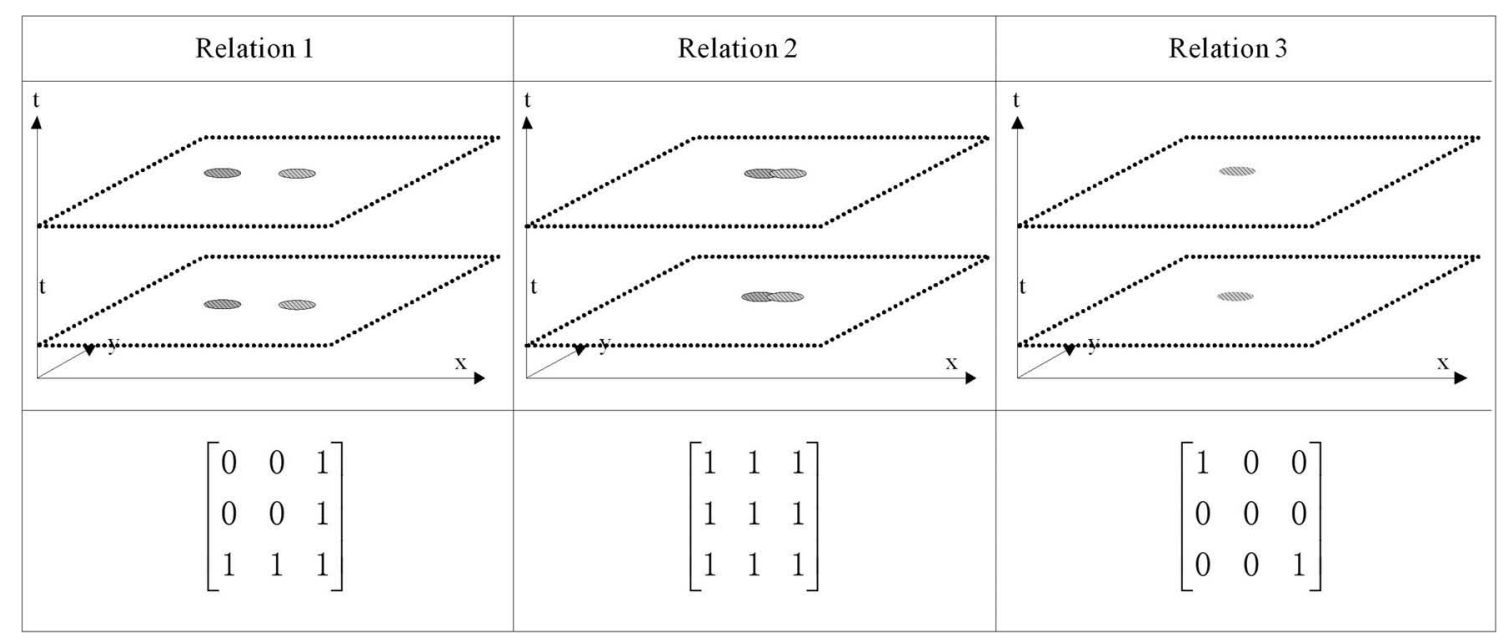

Figure 8 . The three basic topological relationships between two moving fuzzy points. 
It is worth mentioning that the degree of topological relations FPPMeet can be defined by domain experts.

Based on the definition above, we group 3 topological relations shown in Figure 8 into three basic relations. The basic topological relationships, Disjoint, Meet and FPPMeet, correspond to the relations 1, 3 and 2 in Figure 8, respectively.

\section{In the case of $m f$ point/mfregion.}

Similarly to the previous case, we can distinguish 6 different topological relations between moving fuzzy point to moving fuzzy region. The geometric representations and their corresponding intersection matrices are shown in Figure 9.

According to the intuitional meaning of six topological relations, we give the formal definition of six basic topological relationships be- tween moving fuzzy point to moving fuzzy region.

Definition 9. Let $P$ and $R$ denote a moving fuzzy point and a moving fuzzy region, respectively, and let $t$ be a temporal point or a temporal interval. Time domains of $P$ and $R$ are given by $\operatorname{domain}(P)$ and domain $(R)$, respectively. Fuzzy spatio-temporal topological relationships between moving fuzzy point to moving fuzzy region are defined as follows.

$\operatorname{Disjoint}(P, R, t)=\forall t \in \operatorname{domain}(P) \cap \operatorname{domain}(R)$ :

$$
\begin{aligned}
P^{0} \cap_{t} R_{d} & =\varnothing \wedge P^{0} \cap_{t} R_{f}=\varnothing \wedge \partial P \cap_{t} R_{d} \\
& =\varnothing \wedge \partial P \cap_{t} R_{f}=\varnothing .
\end{aligned}
$$

$\boldsymbol{F P R D i s j o i n t}(P, R, t)=\exists t \in \operatorname{domain}(P) \cap \operatorname{domain}(R)$ :

$$
\begin{aligned}
\left(P^{0} \cap_{t} R_{d}\right. & \left.=\varnothing \wedge \partial P \cap_{t} R_{d}=\varnothing\right) \vee \\
\left(P^{0} \cap_{t} R_{d}\right. & =\varnothing \wedge \partial P \cap_{t} R_{d} \\
& =\varnothing \wedge P^{0} \cap_{t} R^{-} \\
& \left.=\varnothing \wedge \partial P \cap_{t} R^{-}=\varnothing\right) .
\end{aligned}
$$

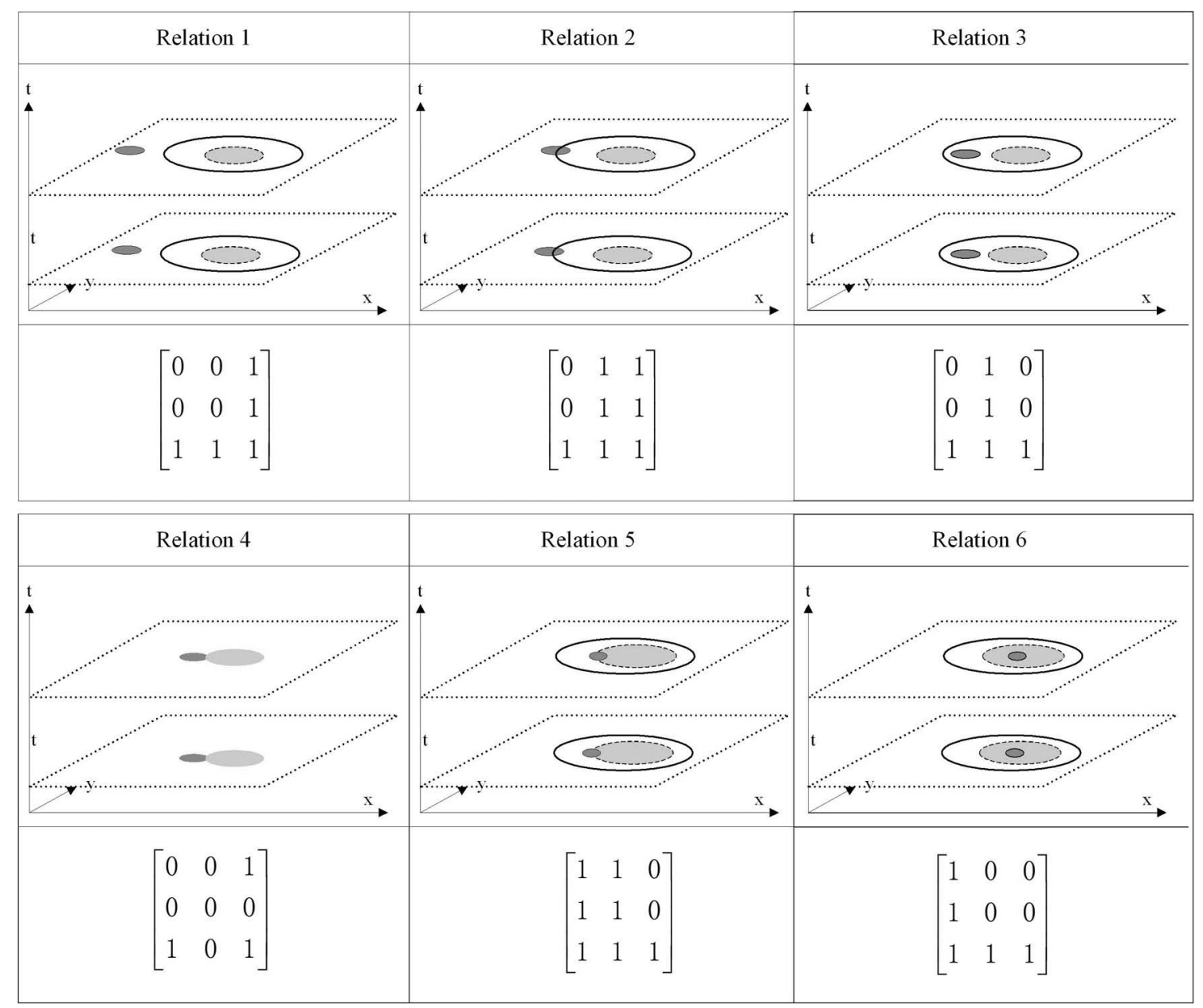

Figure 9. The six relationships between a moving fuzzy point to a moving fuzzy region. 
Inside $(P, R, t)=\forall t \in \operatorname{domain}(P) \cap \operatorname{domain}(R)$ :

$$
\begin{aligned}
P^{0} \cap_{t} R^{-} & =\varnothing \wedge P^{0} \cap_{t} R_{f}=\varnothing \wedge \partial P \cap_{t} R^{-} \\
& =\varnothing \wedge \partial P \cap_{t} R_{f}=\varnothing .
\end{aligned}
$$

FPRInside $(P, R, t)=\exists t \in \operatorname{domain}(P) \cap \operatorname{domain}(R)$ :

$$
\begin{aligned}
\left(P^{0} \cap_{t} R_{d}\right. & =\varnothing \wedge \partial P \cap_{t} R_{d}=\varnothing \wedge P^{0} \cap_{t} R^{-} \\
& \left.=\varnothing \wedge \partial P \cap_{t} R^{-}=\varnothing\right) \vee \\
\left(P^{0} \cap_{t} R^{-}\right. & \left.=\varnothing \wedge \partial P \cap_{t} R^{-}=\varnothing\right) .
\end{aligned}
$$

$\operatorname{Meet}(P, R, t)=\forall t \in \operatorname{domain}(P) \cap \operatorname{domain}(R)$ : $\partial P=\varnothing \wedge R_{f}=\varnothing \wedge P^{0} \cap_{t} R_{d}=\varnothing$.

$\boldsymbol{F P R M e e t}(P, R, t)=\exists t \in \operatorname{domain}(P) \cap \operatorname{domain}(R)$ : FPRDisjoint $(P, R, t) \vee$ FPRInside $(P, R, t)$.

From the definition above, we can see that there are 6 kinds of basic topological relationships between moving fuzzy point to moving fuzzy region. For the Disjoint, we can say that a moving fuzzy point and a moving fuzzy region are always disjoint during a time interval $t$ or at a time point $t$. The relation FPRDisjoint holds if the boundaries and determinate parts of the moving fuzzy point intersect with the outer boundaries or fuzzy parts of the moving fuzzy region. Similarly, the Inside is used to deal with this case that the moving fuzzy point is always inside the moving fuzzy region. The FPRInside holds if the boundary of the moving fuzzy point is located on the boundary of the moving fuzzy regon or the moving point is inside the fuzzy parts of the moving region. Moreover, Meet indicates that the moving fuzzy point certainly meets the moving region when the two objects are crisp and the two crisp parts definitely meet. FPRMeet means that a moving fuzzy point and a moving fuzzy region will be disjoint with some possibility or a moving point will be inside a moving region with some possibility.

Based on the definition above, 6 topological relations shown in Figure 9 can be grouped into six basic relations. The result of grouping these topological relations into the six basic relations is shown in Table 3.

\section{In the case of mfregion/mfregion.}

Similarly to the previous case, 50 different topological relations can be identified between two moving fuzzy regions. The relationships between two moving fuzzy regions are shown in
Table 3. The result of grouping topological relationships into the six basic relations.

\begin{tabular}{|c||c|}
\hline Basic topological relations & $\begin{array}{c}\text { Relations' numbers } \\
\text { in Figure 9 }\end{array}$ \\
\hline \hline Disjoint & 1 \\
\hline FPRDisjoint & 2,3 \\
\hline Meet & 4 \\
\hline FPRMeet & $2,4,5$ \\
\hline Inside & 6 \\
\hline FPRInside & 3,5 \\
\hline
\end{tabular}

Figure 10, and they include the value of matrix plus the support view of 3-dimensional spaces.

In the following, we define formally 16 basic topological relationships between two moving fuzzy regions.

Definition 10. Let $A$ and $B$ denote two moving fuzzy regions, and let $t$ be a temporal point or a temporal interval. Time domains of $A$ and $B$ are given by $\operatorname{domain}(A)$ and $\operatorname{domain}(B)$, respectively. Fuzzy spatio-temporal predicates between two moving fuzzy regions are defined as follows.

$\operatorname{Disjoint}(A, B, t)=\forall t \in \operatorname{domain}(A) \cap \operatorname{domain}(B)$ :

$$
\begin{aligned}
A_{d} \cap_{t} B_{d} & =\varnothing \wedge A_{d} \cap_{t} B_{f}=\varnothing \wedge A_{f} \cap B_{d} \\
& =\varnothing \wedge A_{f} \cap_{t} B_{f}=\varnothing
\end{aligned}
$$

$\boldsymbol{F R R D i s j o i n t}(A, B, t)=\exists \mathrm{t} \in \operatorname{domain}(A) \cap \operatorname{domain}(B)$ :

$\neg \operatorname{Disjoint}(A, B, t) \wedge A_{d} \cap_{t} B_{d}$

$$
=\varnothing \wedge \partial A_{d} \cap_{t} \partial B_{f}=\varnothing
$$

$\operatorname{Meet}(A, B, t)=\forall t \in \operatorname{domain}(A) \cap \operatorname{domain}(B)$ :

$$
\begin{aligned}
A_{d} \cap_{t} B_{f} & =\varnothing \wedge A_{d} \cap_{t} B^{-}=\varnothing \wedge A_{f} \cap B_{d} \\
& =\varnothing \wedge A_{f} \cap_{t} B_{f}=\varnothing \wedge A_{f} \cap_{t} B^{-} \\
& =\varnothing \wedge \mathrm{A}^{-} \cap B_{d}=\varnothing \wedge A^{-} \cap_{t} B_{f}=\varnothing .
\end{aligned}
$$

$\boldsymbol{F R R M e e t}(A, B, t)=\exists t \in \operatorname{domain}(A) \cap \operatorname{domain}(B)$ : $\left(\neg \operatorname{Meet}(A, B, t) \wedge A_{d} \cap_{t} B_{d}=\varnothing \wedge \partial A_{d} \cap_{t} \partial A_{f} \neq \varnothing\right)$ $\vee$ FRRDisjoint $(A, B, t)$.

Inside $(A, B, t)=\exists t \in \operatorname{domain}(A) \cap \operatorname{domain}(B)$ :

$$
\begin{aligned}
A_{d} \cap_{t} B_{f} & =\varnothing \wedge A_{d} \cap_{t} B^{-}=\varnothing \wedge A_{f} \cap B_{f} \\
& =\varnothing \wedge A_{f} \cap_{t} B^{-}=\varnothing
\end{aligned}
$$

FRRInside $(A, B, t)=\exists t \in \operatorname{domain}(A) \cap \operatorname{domain}(B)$ : $\neg$ Inside $(A, B, t) \wedge A_{d} \cap_{t} B^{-}=\varnothing \wedge \partial A_{d} \cap_{t} \partial B_{f}=\varnothing$. 


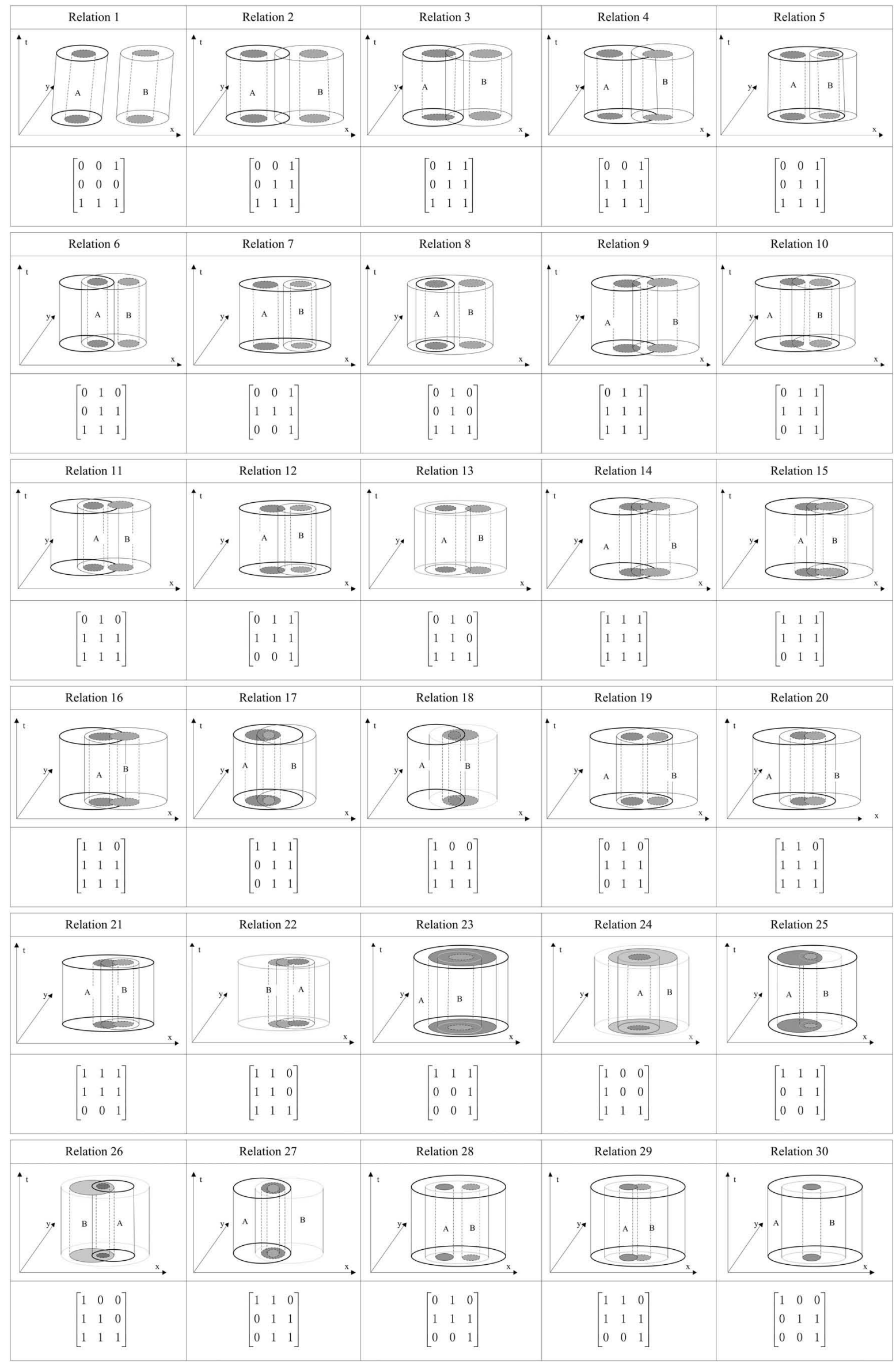




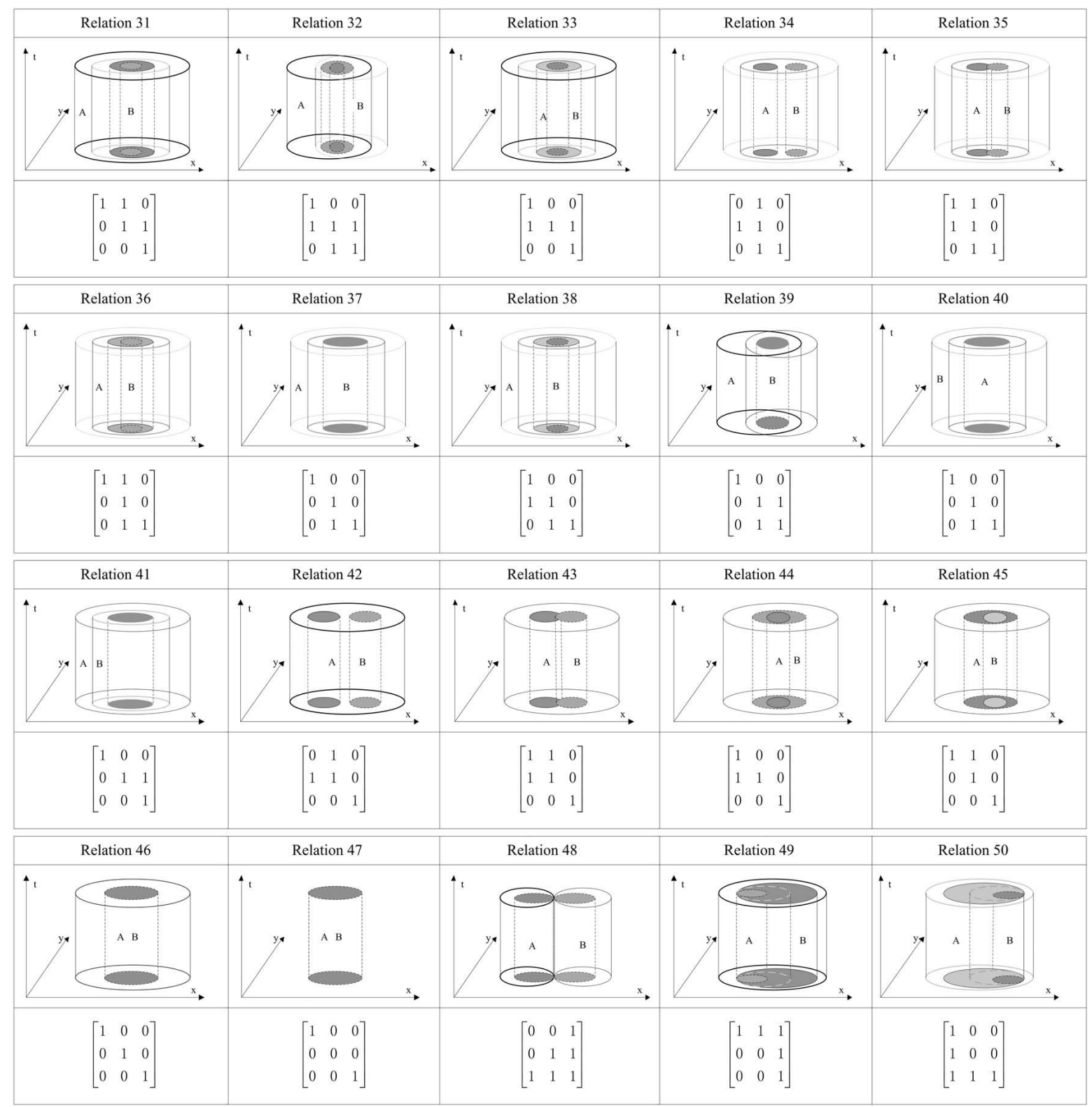

Figure 10. The 50 relationships between two moving fuzzy regions.

Contains $(A, B, t)=$ Inside $(B, A, t)$, FRRContains $(A, B, t)=$ FRRInside $(B, A, t)$.

Coveredby $(A, B, t)=\forall t \in \operatorname{domain}(A) \cap \operatorname{domain}(B)$ :

$$
\begin{aligned}
A_{d} \cap_{t} B_{f} & =\varnothing \wedge A_{d} \cap_{t} B^{-} \\
& =\varnothing \wedge \partial A_{d} \cap_{t} \partial B_{d} \neq \varnothing \wedge A_{f} \cap_{t} B_{f} \\
& =\varnothing \wedge A_{f} \cap_{t} \mathrm{~B}^{-}=\varnothing .
\end{aligned}
$$

FRRCoveredby $(A, B, t)$

$$
=\exists t \in \operatorname{domain}(A) \cap \operatorname{domain}(B):
$$

$\neg$ Coveredby $(A, B, t) \wedge$ FRRInside $(A, B, t)$

$$
\wedge \partial A_{d} \cap_{t} \partial B_{f} \neq \varnothing \text {. }
$$

Covers $(A, B, t)=$ Coveredby $(B, A, t)$, FRRCovers $(A, B, t)=$ FRRCoveredby $(B, A, t)$.

$\operatorname{Equal}(A, B, t)=\forall t \in \operatorname{domain}(A) \cap \operatorname{domain}(B)$ :

$$
\begin{aligned}
A_{f} & =\varnothing \wedge B_{f}=\varnothing \wedge A_{d} \cap_{t} B_{d} \\
& \neq \varnothing \wedge \partial A_{d} \cap_{t} \partial B_{d} \neq \varnothing \wedge A_{d} \cap_{t} \partial B_{d} \\
& \neq \varnothing \wedge \partial A_{d} \cap_{t} B_{d} \neq \varnothing .
\end{aligned}
$$

$\operatorname{FRREqual}(A, B, t)=\exists t \in \operatorname{domain}(A) \cap \operatorname{domain}(B)$ :

$\neg \boldsymbol{E q u a l}(A, B, t) \wedge \boldsymbol{F R R I n s i d e}(A, B, t)$.

Overlap $(A, B, t)=\forall t \in \operatorname{domain}(A) \cap \operatorname{domain}(B)$ : $A_{d} \cap_{t} B_{d} \neq \varnothing \wedge A_{d} \cap_{t} B^{-} \neq \varnothing \wedge A^{-} \cap_{t} B_{d} \neq \varnothing$. 
FRROverlap $(A, B, t)=\exists t \in \operatorname{domain}(A) \cap \operatorname{domain}(B)$ :

$\neg \operatorname{Overlap}(A, B, t) \wedge \neg \operatorname{Disjoint}(A, B, t) \wedge$

$\neg \operatorname{Meet}(A, B, t) \wedge \neg \operatorname{Inside}(A, B, t) \wedge$

$\neg$ Contains $(A, B, t) \wedge \neg$ Coveredby $(A, B, t) \wedge$

$\neg \operatorname{Covers}(A, B, t) \wedge \neg \operatorname{Equal}(A, B, t)$.

For two moving fuzzy regions $A$ and $B$ during a period of time $t$, the Disjoint relationship holds if all components (including determinate and fuzzy parts) are always disjoint with each other. In the exclusion of the case of Disjoint, if the determinate parts of two objects do not intersect and their boundaries also do not intersect, then two moving fuzzy regions will be disjoint with some possibilities. The Meet relationship describes the situation that the inner boundaries of the two objects intersect, but their determinate parts do not intersect and their fuzzy parts do not intersect either. The FRRMeet relationship includes two situations: (i) FRRDisjoint; (ii) in the exclusion of the case of Meet, their determinate parts do not intersect, but their inner boundaries intersect. The relationship Inside holds if the determinate and fuzzy parts of $A$ are inside the determinate of $B$. The relation FRRInside holds if the determinate of $A$ is inside the outer boundaries $\partial B_{f}$ of $B$, but the inner boundaries $\partial A_{d}$ of $A$ do not intersect with the outer boundaries $\partial B_{f}$ of $B$. The relation Contains is symmetric to Inside. Hence, the relations Contains and FRRContains are symmetric to Inside and FRRContains, respectively.

For two moving fuzzy regions $A, B$ during a period of time $t, A$ is definitely covered by $B$ if the determinate and fuzzy parts of $A$ are inside the determinate part of $B$ and the inner boundaries $\partial A_{d}$ of $A$ intersect the inner boundaries $\partial B_{d}$ of $B$. The FRRCoveredby relation holds if the moving fuzzy region $A$ is possibly inside the object $B$ and the inner boundaries $\partial A_{d}$ of $A$ intersect the outer boundaries $\partial B_{f}$ of $B$ in the exclusion of the case of Coveredby relation. Since the relation Coveredby is symmetric to Covers, the Covers and FRRCovers relations are symmetric to Coveredby and FRRCoveredby, respectively. In addition, for the fuzzy spatio-temporal objects $A$ and $B$, if their determinate parts are equal and their fuzzy parts are empty, then we can say that the two objects are definitely equal; if $A$ is possibly inside $B$ in the exclusion of the case of Equal, then we can say that the objects $A$ and $B$ are equal with some possibilities. The Overlap relation between two moving fuzzy regions $A$ and $B$ holds if their determinate parts intersect each other and also intersect their whole exteriors. The relation of FRROverlap describes a situation which excludes 7 definite relations mentioned before.

Based on the definition above, the 50 topological relations shown in Figure 10 can be grouped into 16 basic relations.

The result of grouping these topological relations into 16 basic relations is shown in Table 4 .

Table 4. Grouping topological relationships into 16 basic relations.

\begin{tabular}{|c|c|}
\hline $\begin{array}{l}\text { Basic topological } \\
\text { relations }\end{array}$ & Relations' numbers in Figure 10 \\
\hline Disjoint & 1 \\
\hline FPRDisjoint & $\begin{array}{c}2,3,4,5,6,7,8,9,10,11,12,13,19 \\
28,34,42\end{array}$ \\
\hline Inside & 24 \\
\hline FRRInside & $\begin{array}{l}6,8,11,13,16,18,19,20,22,26,27, \\
28,29,30,31,32,33,34,35,36,37, \\
38,39,40,41,42,43,44,45,46,49\end{array}$ \\
\hline Contains & 23 \\
\hline FRRContains & $\begin{array}{l}5,7,10,12,15,17,19,20,21,25,27, \\
28,29,30,31,32,33,34,35,36,37, \\
38,39,40,41,42,43,44,45,46,50\end{array}$ \\
\hline Equal & 47 \\
\hline FRREqual & $\begin{array}{c}6,8,11,13,16,18,19,20,22,26,27 \\
28,29,30,31,32,33,34,35,36,37, \\
38,39,40,41,42,43,44,45,46\end{array}$ \\
\hline Meet & 48 \\
\hline FRRMeet & $\begin{array}{c}2,3,4,5,6,7,8,9,10,11,12,13,19, \\
28,34,42\end{array}$ \\
\hline Coveredby & 49 \\
\hline FRRCoveredby & $\begin{array}{c}6,8,11,13,16,18,19,20,22,26,27 \\
28,29,30,31,32,33,34,35,36,37, \\
38,39,40,41,42,43,44,45,46\end{array}$ \\
\hline Covers & 50 \\
\hline FRRCovers & $\begin{array}{c}5,7,10,12,15,17,19,20,21,25,27 \\
28,29,30,31,32,33,34,35,36,37, \\
38,39,40,41,42,43,44,45,46\end{array}$ \\
\hline Overlap & 14 \\
\hline FRROverlap & $\begin{array}{l}2,3,4,5,6,7,8,9,10,11,12,13,15 \\
16,17,18,19,20,21,22,25,26,27, \\
28,29,30,31,32,33,34,35,36,37, \\
\quad 38,39,40,41,42,43,44,45,46\end{array}$ \\
\hline
\end{tabular}


In the next section, we will investigate how these basic fuzzy spatio-temporal relationships can be combined into complex ones.

\section{Modeling Complex Topological Relationships}

In this section, we investigate the modeling of complex topological relationships between fuzzy spatio-temporal objects based on basic topological relationships. Section 6.1 gives the formal definition of complex fuzzy spatio-temporal relationships. Section 6.2 elaborates specification of those relationships in the aspects of definition and graphical representation.

\subsection{Formal Definition of Complex Topological Relationships}

In this section, we define some complex topological relationships between fuzzy spatio-temporal objects by means of all basic ones presented in the previous section. In fact, complex topological relationships are the dynamic relations. The complex topological relationships are the sequences of basic topological relationships which hold during a time interval.

In order to well understand the sequence of some basic topological relationships, we first consider a simple scenario presented in [37]. There are two moving objects which are a moving airplane and a moving storm. Firstly, the moving airplane and the moving storm are disjoint over a period of time. Along with their locations change, the airplane meets the strom at some time. Then the airplane enters the storm and is inside the storm for a while. Afterward, the airplane again meets the border of the storm at some time. At last, the airplane and the storm are disjoint again. We call the evolution of a moving airplane with respect to a moving storm during a time interval "a moving airplane crosses a moving storm". According to the scenario, we can observe that the spatio-temporal relation cross can be a sequence of the basic topological relations disjoint, meet, inside, meet, and disjoint. It is important to note that Martin et al. [2] identified so-called instant predicates such as equal, meet, covers, and coveredBy which hold only at a time point. And those relations can only deal with crisp regions, but our work focuses on the fuzzy spatio-temporal objects such as moving fuzzy point, moving fuzzy line, and moving fuzzy region which change over a period of time. Hence, generally, in our work, all the basic and complex fuzzy spatio-temporal relationships are used for a time interval. In the following, we give a formal definition of complex topological relationships between fuzzy spatio-temporal objects.

Definition 11 (Complex Topological Relationships). Let $\alpha, \beta \in\{$ mfpoint, mfline, mfregion $\}$ be two fuzzy spatio-temporal objects and let $I=$ $\left[I_{S}, I_{E}\right]$ be a whole time interval containing the start time $I_{S}$ and the end time $I_{E}$; such that $I$ is the intersection of the time domain over $\alpha$ and $\beta, I=$ timeDomain $(\alpha) \cap$ timeDomain $(\beta)$. Let $P$ be a finite set of basic fuzzy spatio-temporal relationships defined in Section 5.2, $P=\left\{p_{1}, p_{2}\right.$, $\left.\ldots, p_{n}\right\}, n \geq 2$. A complex fuzzy spatio-temporal relationship $C$ between $\alpha$ and $\beta$ is defined as:

$$
\begin{aligned}
C & \stackrel{\text { def }}{=} \exists t_{1}, t_{2}, \ldots, t_{n} \in I \wedge t_{1}<t_{2}<\ldots<t_{n} \\
& \wedge p_{1}\left(\alpha, \beta, t_{1}\right) \wedge p_{2}\left(\alpha, \beta, t_{2}\right) \\
& \wedge \ldots \wedge p_{n}\left(\alpha, \beta, t_{n}\right) .
\end{aligned}
$$

According to the above definition, we can observe that a complex fuzzy spatio-temporal relation is constructed from basic topological relationship mentioned before. In fact, a complex topological relationship is a motion verb which represents the change of fuzzy spatio-temporal topological relations over a period of time. It should be noted that one motion verb can represent at least two basic fuzzy spatio-temporal relations at one specified time. This is the reason why we set $n \geq 2$ in the above definition. In addition, we can know that sequential composition satisfies the associativity. As a result, we can easily define complex fuzzy spatio-temporal relations by successively composing some simple topological relations we have obtained. For example, the complex topological relation cross can be composed by the enter and its corresponding relation leave. Hence, the above definition can also be written in the following form.

$$
\begin{aligned}
C & \stackrel{\text { def }}{=} \exists t_{1}, t_{2}, \ldots, t_{n} \in I \wedge t_{1}<t_{2}<\ldots<t_{n} \\
& \wedge C_{1}\left(\alpha, \beta, t_{1}\right) \wedge C_{2}\left(\alpha, \beta, t_{2}\right) \\
& \wedge \ldots \wedge C_{n}\left(\alpha, \beta, t_{n}\right) .
\end{aligned}
$$


Here, $C_{1}, C_{2}, \ldots, C_{n}$ denote the simple topological relations we have obtained.

For the two forms, we can observe that either basic fuzzy spatio-temporal relation or simple relation hold before or after another relation. In other words, basic or simple fuzzy spatio-temporal relations start or end with another ones. For example, in the first form, the relation $p_{2}$ (during the time $t_{2}$ ) holds after the end of $p_{1}$ (during the time $\mathrm{t} 1$ ); in the second form, the relation $C_{1}$ (during the time $t_{1}$ ) ends with $C_{2}$ (during the time $t_{2}$ ). As we have mentioned, the temporal interval comprises many basic temporal intervals of basic fuzzy spatio-temporal relations. The start is the beginning of first basic fuzzy spatio-temporal relation, and the end is the end of the last relation. Hence, we can define a temporal composition to combine basic or simple fuzzy spatio-temporal relations.

Definition 12 (Temporal Composition). Let $\alpha$, $\beta$ be two fuzzy spatio-temporal objects. Let $P$ and $Q$ be basic or simple fuzzy spatio-temporal relations, and let the symbol of $\triangleright$ be the temporal composition operator. Then,

$$
\begin{aligned}
P \triangleright C \stackrel{\text { def }}{=} \exists t_{1}, t_{2} \in I \wedge t_{1}<t_{2}: \\
P\left(\alpha, \beta, t_{1}\right) \wedge Q\left(\alpha, \beta, t_{2}\right)
\end{aligned}
$$

The definition describes temporal compositions of basic fuzzy spatio-temporal relations which are denoted by the operator $\triangleright$. The aim of the temporal composition is to represent evolution of fuzzy spatio-temporal objects concisely. The operator can make full use of the definitions of basic relations mentioned above to denote the evolution. On the other hand, the definition is conducive to establishing alternating sequences of fuzzy spatio-temporal relations. Notice that, temporal compositions can only hold if there are at least two fuzzy spatio-temporal relations or alternating sequences. For instance, consider two moving fuzzy points $o_{1}$ and $o_{2}$. We can describe a sequence of basic fuzzy spatio-temporal relations during some time interval $I$ :

\section{Disjoint $\triangleright$ Meet $\triangleright$ Disjoint .}

In the next section, we will be in accordance with the three cases to define the complex fuzzy spatio-temporal relation specifications in detail. Due to the lack of space, we only give limited geometric representations of fuzzy spatio-temporal topological relationships.

\subsection{Modeling of Complex Topological Relationships}

\section{In the case of mfpoint/mfpoint.}

As mentioned in Section 5.2, we have obtained three basic topological relations, such as Disjoint, Meet and FPPMeet. Figure 11 shows the evolution of those 3 basic relations.

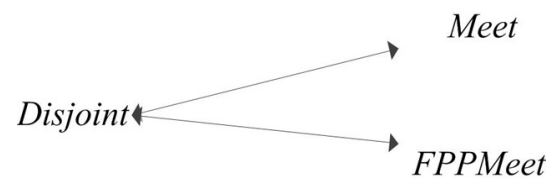

Figure 11. The evolution of 3 basic relations in the case of mfpoint/mfpoint.

According to the figure above, we can obtain 2 possible development paths, from Disjoint to Meet to Disjoint, and Disjoint to FPPMeet to Disjoint. As a result, we define two new vocabularies to represent those two paths. Formally ( $\alpha$ and $\beta$ denote two moving fuzzy points):

Encounter $(\alpha, \beta, I)=$ Disjoint $\triangleright$ Meet $\triangleright$ Disjoint . FPPEncounter $(\alpha, \beta, I)$

$$
=\text { Disjoint } \triangleright \text { FPPMeet } \triangleright \text { Disjoint } \text {. }
$$

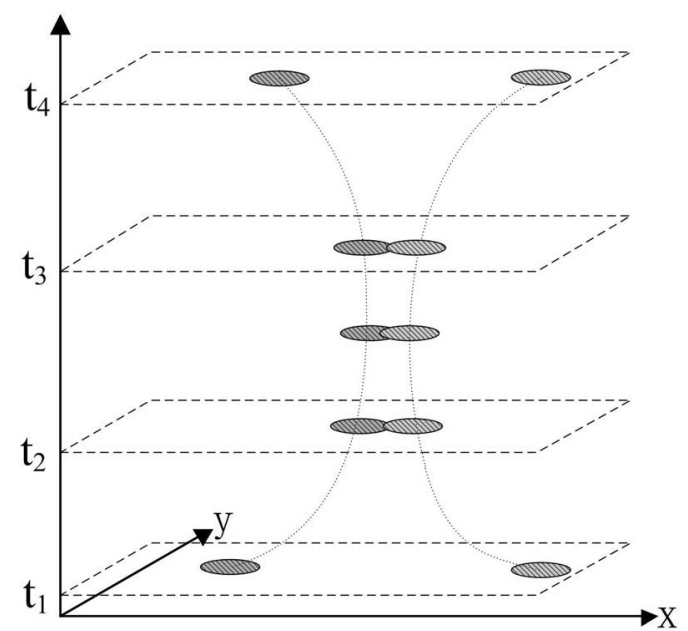

Figure 12. An illustration of Encounter evolution.

For example, we give graphical representations of Encounter evolution between two moving fuzzy points, as shown in Figure 12. From the figure, we can observe that the relation between 
two moving fuzzy points remains Encounter from $t_{1}$ to $t_{4}$. More precisely, from $t_{1}$ to $t_{2}$, the relation is Disjoint; from $t_{2}$ to $t_{3}$, the relation is FPPMeet; from $t_{3}$ to $t_{4}$, the relation is Disjoint; thus, from $t_{1}$ to $t_{4}$, the new relation is defined as Encounter. For the reason of space, the graphical representation of FPPEncounter is not given.

2. In the case of $m f$ point/mfregion.

In the case of $m f$ point/mfregion, there are 8 new complex relationships to be defined as follows:

Touch $(\alpha, \beta, I)=$ Disjoint $\triangleright F P R D$ isjoint $\triangleright$ Meet $\triangle$ FPRDisjoint $\triangle$ Disjoint.

$\boldsymbol{F P R T o u c h}(\alpha, \beta, \mathrm{I})=$ Disjoint $\triangleright$ FPRMeet

$$
\triangleright \text { Disjoint. }
$$

Enter $(\alpha, \beta, I)=$ Disjoint $\triangleright F P R$ Disjoint $\triangleright$ Meet $\triangle$ FPRInside $\triangleright$ Inside.

FPREnter $(\alpha, \beta, I)=$ Disjoint $\triangle F P R$ Disjoint $\triangle$ Meet $\triangle$ FPRInside.

Leave $(\alpha, \beta, I)=$ Inside $\triangleright$ FPRInside $\triangleright$ Meet $\triangleright$ FPRMeet $\triangleright$ Disjoint.

FPRLeave $(\alpha, \beta, I)=$ Inside $\triangle F P R I n$ side $\triangleright$ Meet $\triangle F P R M e e t \triangle F P R D i s j o i n t$.

$\operatorname{Cross}(\alpha, \beta, I)=$ Enter $\triangleright$ Leave,

$\boldsymbol{F P R C r o s s}(\alpha, \beta, I)=$ Enter $\triangleright$ Leave.

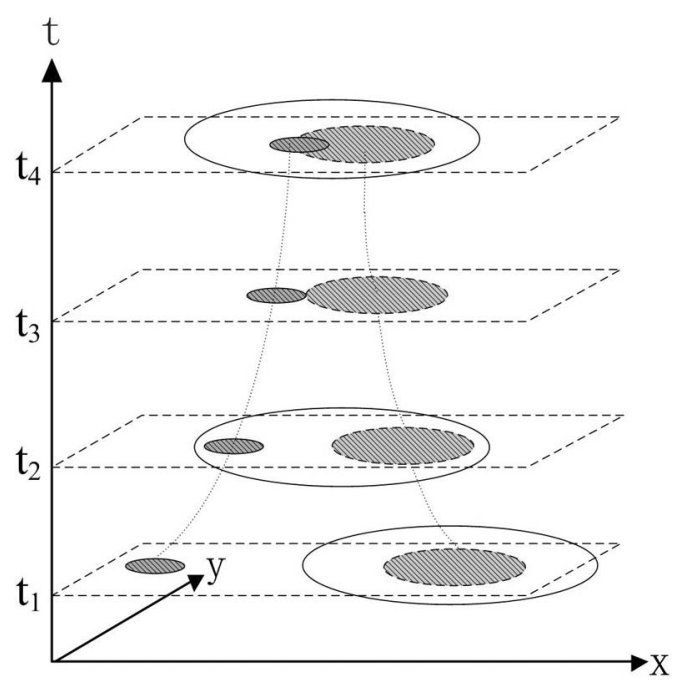

Figure 13. An illustration of FPREnter evolution.

For example, we give graphical representations of FPREnter evolution between a moving fuzzy point to a moving fuzzy region, as shown in
Figure 13. From the figure, we can observe that the development of a moving fuzzy point and a moving fuzzy region includes four states, Disjoint, FPRDisjoint, Meet, and FPRInside. As a result, the whole evolution of them remains FPREnter during the time interval $\left[t_{1}, t_{4}\right]$. Due to the lack of space, the graphical representations of other fuzzy spatio-temporal relations are not given, which are similar to the case of the predicate FPREnter.

\section{In the case of mfregion/mfregion.}

In the case of mfregion/mfregion, there are 16 kinds of complex topological relations to be defined as follows:

Touch $(\alpha, \beta, I)=$ Disjoint $\triangleright F P R D$ isjoint $\triangleright$ Meet $\triangle F P R$ Disjoint $\triangle$ Disjoint.

$\boldsymbol{F P R T o u c h}(\alpha, \beta, \mathrm{I})=$ Disjoint $\triangleright$ FPRMeet $\triangleright$ Disjoint.

Into $(\alpha, \beta, I)=$ Meet $\triangleright$ Overlap $\triangle F R R$ CoveredBy $\triangleright$ CoveredBy.

FRRInto $(\alpha, \beta, I)=$ Meet $\triangleright$ Overlap $\triangle F R R$ Covered By.

OutOf $(\alpha, \beta, I)=$ CoveredBy $\triangleright F R R$ CoveredBy $\triangle$ Overlap $\triangleright$ Meet.

FRROutOf $(\alpha, \beta, I)=$ CoveredBy $\triangle F R R$ CoveredBy $\triangle$ Overlap $\triangle$ FRRMeet.

Enter $(\alpha, \beta, I)=$ Disjoint $\triangleright F R R$ Disjoint $\triangleright$ Into $\triangle$ FRRInside $\triangleright$ Inside.

FRREnter $(\alpha, \beta, I)=$ Disjoint $\triangleright F R R$ Disjoint $\triangle$ Into $\triangleright$ FRRInside.

Leave $(\alpha, \beta, I)=$ Inside $\triangleright$ FRRInside $\triangleright$ OutOf

$\triangle$ FRRDisjoint $\triangle$ Disjoint.

FRRLeave $(\alpha, \beta, I)=$ Inside $\triangleright$ FRRInside $\triangleright$ OutOf $\triangleright$ FRRDisjoint.

$\operatorname{Cross}(\alpha, \beta, I)=$ Enter $\triangleright$ Leave,

$\operatorname{FRRCross}(\alpha, \beta, I)=$ Enter $\triangleright F R R L e a v e$.

$\operatorname{Melt}(\alpha, \beta, I)=$ Disjoint $\triangle F R R$ Disjoint $\triangleright$ Meet $\triangle$ Overlap $\triangleright$ FRREqual $\triangleright$ Equal.

FRRMelt $(\alpha, \beta, I)=$ Disjoint $\triangle F R R$ Disjoint $\triangleright$ Meet $\triangle$ Overlap $\triangle$ FRREqual.

Separate $(\alpha, \beta, I)=$ Equal $\triangleright$ FRREqual

$\triangle$ Overlap $\triangleright$ Meet

$\triangleright$ FRRDisjoint $\triangleright$ Disjoint.

FRRSeparate $(\alpha, \beta, I)=$ Equal $\triangleright$ FRREqual

$\triangleright$ Overlap $\triangleright$ Meet

$\triangleright$ FRRDisjoint. 
For example, Figure 14 gives an illustration of Into evolution between two moving fuzzy regions. Due to the lack of space, the other relations in the case of $m$ fregion/mfregion are not given.

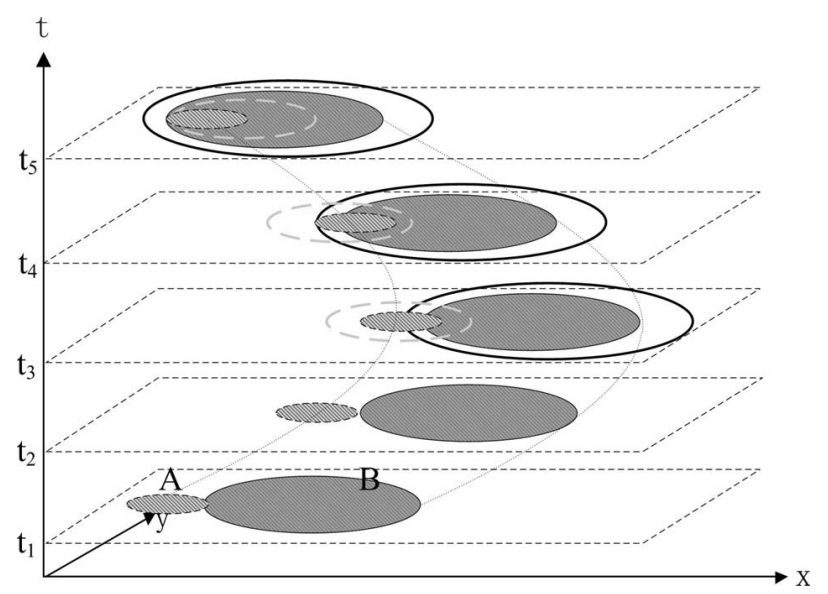

Figure 14. An illustration of Into evolution.

Up to now, we have constructed a model of complex topological relationships between fuzzy spatio-tempral objects. In the next section, we will use these topological relationships to query fuzzy spatio-temporal information in the context of spatio-temporal database.

\section{Fuzzy Spatio-Temporal Queries}

In this section, we discuss how to use basic and complex topological relationships we have defined in sections 5 and 6 to achieve fuzzy spatio-temporal queries.

Querying fuzzy spatio-temporal objects through topological relationships is one of the basic tasks in spatio-temporal databases or GIS. Currently, the existing database query languages such as SQL cannot answer fuzzy spatio-temporal queries because they cannot support fuzzy spatio-temporal query operators. Our proposed model can well address the problem of fuzzy spatio-temporal queries. More precisely, we use the basic and complex topological relationships as fuzzy spatio-temporal query operators. This is the first step. We integrate these operators into spatio-temporal database and query language. This is the second step.

Now, we illustrate fuzzy spatio-temporal queries according to the following three cases. For each case, we give an application scenario.

\section{In the case of $m f$ point/mfpoint.}

In this case, we consider an application of multimedia video. It focuses on complex topological relationships between two moving fuzzy points. Assume that persons have the following relation schema:

Persons (id: integer, name: string, trajectory: mfpoint)

Now, we consider a fuzzy spatio-temporal query problem: Find all persons that may become the witnesses of criminal Tom during the time interval [2:00am, 5:00am]. A fuzzy spatio-temporal query can be expressed as follows:

SELECT $\mathrm{p} 1$. id

FROM persons p1, person $\mathrm{p} 2$

WHERE FPPEncounter (p1. trajectory, p2. trajectory, 2:00:00, 5:00:00) AND p2. Name $=$ 'Tom'.

The query demonstrates the use of topological relation FPPEncounter and retrieves some moving fuzzy points which may become the witnesses of criminal Tom during the time interval [2:00am, 5:00am]. In fact, the complex relation FPPEncounter corresponds to a degree of membership with $[0,1)$.

\section{In the case of mfpoint/mfregion.}

In this case, we consider an application of maritime traffic. It focuses on complex topological relationships between a moving fuzzy point and a moving fuzzy region. There are a lot of ships which are waiting in the queue for crossing Qiongzhou Strait every day. Here, the sailing ships can be considered as moving fuzzy points since their positions may change from time to time, and Qiongzhou Strait can be considered as fuzzy region since its boundaries are fuzzy. It should be noted that fuzzy region is a special case of moving fuzzy region because the locations of fuzzy region cannot change over time. Assume that ships and straits have the following relation schemas:

Ships (id: string, trajectory: mfpoint)

Straits (id: string, name: string, area: fregion)

Now, we give a fuzzy spatio-temporal query problem: Find all ships that may have crossed 
the Qiongzhou Strait from 2015-05-12 08:00 am to 2015-05-13 08:00 am. From the query problem, it is not hard to see that we need integrate complex fuzzy spatio-temporal relationship (operator) FPRCross into query language. Next, we give a fuzzy spatio-temproal query expressed in an SQL-like query language.

SELECT Ships. id

FROM Ships, Straits

WHERE FPRCross (Ships. trajectory, Straits. area, 2015-05-12 08:00:00, 2015-05-13 08:00:00) AND Straits. name = "Qiongzhou Strait".

The query demonstrates the use of complex topological relationship FPRCross and retrieves some moving fuzzy points which have possibly crossed the Qiongzhou Strait from 2015-05-12 08:00 am to 2015-05-13 08:00 am.

We consider a fuzzy spatio-temoral query problem: Find all ships that have entered the Qiongzhou Strait from 2015-05-14 08:00 am to 2015-05-14 09:00 am. Similar to the previous problem, the query can be expressed as follows:

SELECT Ships. id

FROM Ships, Straits

WHERE Enter (Ships. trajectory, Straits. area, 2015-05-14 08:00:00, 2015-05-14 09:00:00)

AND Straits. name = "Qiongzhou Strait".

The query demonstrates the use of complex topological relationship Enter and retrieves some moving fuzzy points which have entered the Qiongzhou Strait from 2015-05-14 08:00:00 to 2015-05-14 09:00:00.

\section{In the case of mfregion/mfregion.}

In this case, we consider a scenario about an application of meteorology. It focuses on complex topological relationships between two moving fuzzy regions.

We assume that hurricanes are moving fuzzy regions since their locations can change over time and their borders are fuzzy. We assume that hurricanes have the following relation schema:

Hurricanes (id: string, name: string, trajectory: mfregion)
Now, we give a fuzzy spatio-temporal query problem: Find all hurricanes that have definitely melted the Meari hurricane from 2011-06-24 24:00 to 2011-06-27 24:00. According to the definition of complex topological relations between two moving fuzzy regions in Section 6.2, we choose Melt to query the problem. Next, we give a fuzzy spatio-temporal query expressed in an SQL-like query language.

SELECT h1. name

FROM Hurricanes h1, Hurricanes h2

WHERE Melt (h1. trajectory, h2. trajectory, 2011-06-24 24:00:00, 2011-06-27 24:00:00)

AND h2. name = "Meari".

The query demonstrates the use of topological relation Melt and retrieves some moving fuzzy regions which definitely melted the Meari hurricane from 2011-06-24 24:00 to 2011-06-27 24:00. In fact, the complex relation Melt corresponds to a degree of membership 1.

Consider a fuzzy spaito-temoral query problem: Find all hurricanes that have possibly leaved the Meari hurricane from 2011-06-24 24:00 to 2011-06-27 24:00. Similar to the previous problem, the query can be expressed as follows:

SELECT h1. name

FROM Hurricanes h1, Hurricanes h2

WHERE FRRLeave (h1. trajectory, h2. trajectory, 2011-06-24 24:00:00, 2011-06-27 24:00:00) AND h2. name = "Meari".

As a result, the query can get all hurricanes identifiers that have fuzzy spatio-temporal topological relation FRRLeave with Meari hurricane from 2011-06-24 24:00 to 2011-06-27 24:00.

\section{Conclusion and Future Work}

In this paper, we present the results of a modeling study on topological relationships between moving fuzzy point to moving fuzzy point, moving fuzzy point to moving fuzzy region, and moving fuzzy region to moving fuzzy region. Firstly, based on fuzzy spatial data types, we define fuzzy spatio-temporal objects as moving fuzzy points, moving fuzzy lines, and moving fuzzy regions in three-dimensional space. Sec- 
ondly, based on the 9-intersection model, we further model fuzzy spatio-temporal topological relations between two fuzzy spatio-temporal objects. By eliminating those relations that are not realized, we can identify a total of 3 topological relations between two moving fuzzy points, 6 topological relations between a moving fuzzy point to a moving fuzzy region, and 50 topological relations between two moving fuzzy regions. For each topological relationship, the corresponding graphical representation and the intersection matrix are also given. Moreover, we define 4 basic relations between two moving fuzzy points, 6 basic relations between moving fuzzy point to moving fuzzy region, and 16 basic relations between two moving fuzzy regions. We also group topological relationships identified into basic relations. In order to describe the evolutions of basic topological relations between fuzzy spatio-temporal objects over time, we give a model of complex spatio-temporal relationships based on basic topological relations. Concretely speaking, complex fuzzy spatio-temproal relations are the sequences of basic topological relations, which hold at the beginning and end of temporal interval. As a result, we define one complex relation between two moving fuzzy points, 8 complex relations between moving fuzzy point to moving fuzzy region, and 16 complex relations between two moving fuzzy regions. Finally, we give a series of application examples to demonstrate fuzzy spatio-temporal queries.

In the future, we will address an experiment to illustrate the effectiveness and efficiency of the proposed method for fuzzy spatio-temporal query and conduct experiment over a real data set in terms of usefulness, effectiveness and efficiency. Our proposed model focuses on temporal change of fuzzy spatial objects and evolution of spatial topological relations. The existing model implementation for fuzzy/vague spatial objects, such as [38], cannot be applied directly to our model. Thus, in the future, we will implement our proposed model in GIS spatio-temporal database.

\section{Acknowledgments}

The work is supported by the National Natural Science Foundation of China (61370075, 61572118 and 61672139).

\section{References}

[1] S. Ribaric and T. Hrkac, "A Knowledge Representation and Reasoning Based on Petri Nets with Spatio-Temporal Tokens", in International Conference on Computer As a Tool (EUROCON2007), pp. 793-800, 2007.

http://dx.doi.org/10.1109/EURCON.2007.4400398

[2] M. Erwig and M. Schneider, "Spatio-Temporal Predicates", IEEE Transactions on Knowledge and Data Engineering, vol. 14, no. 4, pp. 881-901, 2002.

https://doi.org/10.1109/tkde.2002.1019220

[3] M. Erwig and M. Schneider, "STQL - A Spatio-Temporal Query Language", Springer US, pp. 105-126, 2002.

http://dx.doi.org/10.1007/978-1-4615-1149-6_6

[4] Y. Theodoridis et al., "Specifications for Efficient Indexing in Spatiotemporal Databases", in Proceedings of the 10th International Conference on Scientific and Statistical Database Management, pp. 123-132, 1998.

https://doi.org/10.1109/ssdm.1998.688117

[5] S. Grumbach et al., "Spatio-Temporal Data Handling with Constraints", GeoInformatica, vol. 5, no. 1, pp. 95-115, 2001.

https://doi.org/10.1145/288692.288712

[6] N. R. Brisaboa et al., "Constraints in Spatio-Temporal Databases: A Proposal of Classication", in Proceedings of the 3rd International Workshop on Evaluation of Modeling Methods in Systems Analysis and Design, 1998.

[7] M. F. Worboys, "A Unified Model for Spatial and Temporal Information", The Computer Journal, vol. 37, no. 1, pp. 26-34, 1994.

http://dx.doi.org/10.1093/comjnl/37.1.26

[8] M. Schneider, "Moving Objects in Databases and GIS: State-of-the-Art and Open Problems", Springer-Verlag, pp. 169-188, 2009.

http://dx.doi.org/10.1007/978-3-540-88244-2_12

[9] P. Mulle, "Space-Time as a Primitive for Space and Motion", in Proceedings of the International Conference on Formal Ontology in Information Systems, pp. 63-76, 1998.

[10] A. Miene and U. Visser, "Interpretation of SpatioTemporal Relations in Real-Time and Dynamic Environments", Springer Berlin Heidelberg, 2002. https://doi.org/10.1007/3-540-45603-1_57

[11] A. Sözer et al., "Modeling and Querying Fuzzy Spatiotemporal Databases", Information Sciences, vol. 178, no. 19, pp. 3665-3682, 2008.

http://dx.doi.org/10.1016/j.ins.2008.05.034

[12] K. Liu and W. Shi, "Computing Fuzzy Topological Relations of Spatial Objects Based on Induced Fuzzy Topology", International Journal of Geographical Information Science, vol. 20, no. 8, pp. 857-883, 2006.

https://doi.org/10.1080/13658810600711345 
[13] A. Bassiri et al., "Spatio-Temporal Object Modeling in Fuzzy Topological Space", The International Archives of the Photogrammetric, Remote Sensing and Spatial Information Sciences, vol. 37, no. B2, pp. 131-134, 2008.

[14] Z. M. Ibrahim and A. Y. Tawfik, "Spatio-Temporal Reasoning for Vague Regions", Springer Berlin Heidelberg, pp. 308-321, 2004. https://doi.org/10.1007/978-3-540-24840-8 22

[15] D. Pfoser and C. S. Jensen, "Capturing the Uncertainty of Moving-Object Representations", in Proceedings of the 6th International Symposium on the Advances in Spatial Databases, pp. 111131, 1999. https://doi.org/10.1007/3-540-48482-5_9

[16] S. Ribaric and T. Hrkac, "A Model of Fuzzy Spatio-Temporal Knowledge Representation and Reasoning Based on High-Level Petri Nets", Information Systems, vol. 37, no. 3, pp. 238-256, 2012. https://doi.org/10.1016/j.is.2011.09.010

[17] A. Bassiri and A. A. Alesheikh, "Spatio-Temporal Topological Relationships Based on Rough Set", in J. Holub and J. Zdarek (Eds.), in Proceedings of the 7th IEEE International Conference on Cognitive Informatics (ICCI 2008), pp. 175-180, 2008. https://doi.org/10.1109/coginf.2008.4639166

[18] L. Bai et al., "Fuzzy Spatio-Temporal Data Modeling and Operators in XML", Applied Artificial Intelligence, vol. 29, no. 3, pp. 259-282, 2015.

[19] J. Le Yaouanc and J. Poli, "A Fuzzy Spatio-Temporal-Based Approach for Activity Recognition", Springer Berlin Heidelberg, pp. 314-323, 2012. https://doi.org/10.1007/978-3-642-33999-8_37

[20] L. Bai et al., "Modeling Topological Relations between Fuzzy Spatiotemporal Regions Over Time", Theoretical Computer Science, in Proceedings of 2012 IEEE International Conference on Fuzzy Systems (FUZZ-IEEE), pp. 1-8, 2012. https://doi.org/10.1109/fuzz-ieee.2012.6251184

[21] B. Lei et al., "Reasoning the Spatiotemporal Relations between Time Evolving Indeterminate Regions", in Proceedings of the 4th International Conference on Advances in Machine Learning and Cybernetics (ICLMC 2006), pp. 448-458, 2006.

https://doi.org/10.1007/11739685_47

[22] M. J. Egenhofer and R. D. Franzosa, "Point-Set Topological Spatial Relations", International Journal of Geographical Information Systems, vol. 5, no. 2, pp. 161-174, 1991. https://doi.org/10.1080/02693799108927841

[23] H. Liu and M. Schneider, "Querying Moving Objects with Uncertainty in Spatio-Temporal Databases", in Proceedings of the 16th International Conference on Database Systems for Advanced Applications (DASFAA 2011), pp. 357-371, 2011. http://dx.doi.org/10.1007/978-3-642-20149-3 27
[24] L. A. Zadeh, "Fuzzy Sets", Information Control, vol. 8, no. 3, pp. 338-353, 1965.

http://dx.doi.org/10.1016/S0019-9958(65)90241-X

[25] T. Lukasiewicza and U. Straccia, "Managing Uncertainty and Vagueness in Description Logics for the Semantic Web", Web Semantics: Science, Services and Agents on the World Wide Web, vol. 6, no. 4, pp. 291-308, 2008.

http://dx.doi.org/10.1016/j.websem.2008.04.001

[26] D. Papadias and Y. Theodoridis, "Spatial Relations, Minimum Bounding Rectangles, and Spatial Data Structures", International Journal on Geographic Information Science, vol. 11, no. 2, pp. 111-138, 1997.

http://dx.doi.org/10.1080/136588197242428

[27] D. A. Randell et al., "A Spatial Logic Based on Regions and Connection", in Proceedings of the $3 r d$ International Conference on Knowledge Representation and Reasoning (KR-92), pp. 165-176, 1992.

[28] K. S. Na et al., "Multimedia Information Retrieval Based on Spatiotemporal Relationships Using Description Logics for the Semantic Web", International Journal of Intelligent Systems, vol. 21, no. 7, pp. 679-692, 2006. http://dx.doi.org/10.1002/int.20153

[29] M. B. Vilain and H. A. Kautz, "Constraint Propagation Algorithms for Temporal Reasoning", in Proceedings of the 5th National Conference on Artificial Intelligence (AAAI-86), pp. 377-382, 1986.

[30] J. F. Allen, "Towards a General Theory of Action and Time", Artificial Intelligence, vol. 23, no. 2, pp. 123-154, 1984. http://dx.doi.org/10.1016/0004-3702(84)90008-0

[31] J. F. Allen, "Maintaining Knowledge about Temporal Intervals", Communications of the ACM, vol. 26, no. 11, pp. 832-843, 1983. http://dx.doi.org/10.1145/182.358434

[32] M. J. Egenhofer and D. M. Mark, "Modelling Conceptual Neighbourhoods of Toplogical Line-Region Relations", International Journal of Geographical Information Systems, vol. 9, no. 5, pp. 555-565, 1995. http://dx.doi.org/10.1080/02693799508902056

[33] M. Schneider, "Spatial Plateau Algebra for Implementing Fuzzy Spatial Objects in Databases and GIS: Spatial plateau data types and operations", Applied Soft Computing, vol. 16, pp. 148-170, 2014.

https://doi.org/10.1016/j.asoc.2013.11.021

[34] A. Pauly and M. Schneider, "Vague Spatial Data Types, Set Operations, and Predicates", in Proceedings of the Eighth East-European Conference on Advances in Databases and Information Systems, pp. 379-392, 2004. https://doi.org/10.1007/978-3-540-30204-9_26 
[35] X. M. Tang et al., "Modeling of Fuzzy Spatial Objects and their Topological Relations", in Proceedings of the 2nd Symposium on Spatial Data Quality (SDQ), pp. 34-50, 2003.

[36] L. Forlizzi et al., "A Data Model and Data Structures for Moving Objects Databases", in Proceedings of ACM SIGMOD International Conference on Management of Data, pp. 319-330, 2000. http://dx.doi.org/10.1145/342009.335426

[37] M. Schneider, "Evaluation of Spatio-Temporal Predicates on Moving Objects", in Proceedings of the 21st International Conference on Data Engineering (ICDE 2005), pp. 516-517, 2005. http://dx.doi.org/10.1109/ICDE.2005.62

[38] S. Davari and N. Ghadiri, "Spatial Database Implementation of Fuzzy Region Connection Calculus for Analysing the Relationship of Diseases", in Proceedings of the 23rd Iranian Conference on Electrical Engineering, pp. 734-739, 2015. http://dx.doi.org/10.1109/IranianCEE.2015.7146310

Received: December 2015 Revised: November 2016 Accepted: November 2016
Contact addresses:

Haitao Cheng

School of Computer Science and Engineering

Northeastern University

Shenyang 110819

China

e-mail: haitaneu@126.com

Fu Zhang

School of Computer Science and Engineering

Northeastern University

Shenyang 110819

China

e-mail: zhangfu@ise.neu.edu.cn

Haitao Cheng is currently a PhD candidate in School of Computer Science and Engineering at Northeastern University, China. His current research interests include spatio-temporal data management, description logics, ontology and the Semantic Web.

Fu ZHANG received his PhD degree from Northeastern University, China. He is currently an associate professor in School of Computer Science and Engineering at Northeastern University, China. He has authored more than 30 refereed international journals and conference papers. His research work is published in high quality international conferences (e.g., CIKM and DEXA) and in highly cited international journals (e.g., Fuzzy Sets and Systems, Knowledge-Based Systems, and CIT. Journal of Computing and Information Technology). He has also authored two monographs published by Springer. His current research interests include ontology management, the Semantic Web, and knowledge representation and reasoning. 